\title{
Higher-Dimensional Stick Percolation
}

\author{
Erik I. Broman ${ }^{1}$
}

Received: 30 November 2020 / Accepted: 10 November 2021 / Published online: 7 December 2021

(c) The Author(s) 2021

\begin{abstract}
We consider two cases of the so-called stick percolation model with sticks of length $L$. In the first case, the orientation is chosen independently and uniformly, while in the second all sticks are oriented along the same direction. We study their respective critical values $\lambda_{c}(L)$ of the percolation phase transition, and in particular we investigate the asymptotic behavior of $\lambda_{c}(L)$ as $L \rightarrow \infty$ for both of these cases. In the first case we prove that $\lambda_{c}(L) \sim L^{-2}$ for any $d \geq 2$, while in the second we prove that $\lambda_{c}(L) \sim L^{-1}$ for any $d \geq 2$.
\end{abstract}

Keywords Continuum-percolation $\cdot$ Stick percolation $\cdot$ Scaling exponent

Mathematics Subject Classification Primary 60K35 · Secondary 60D05

\section{Introduction}

The two-dimensional Poisson stick model is a classical continuum-percolation model. The first paper focused on this model was [9], although earlier papers such as [2] and [4] included this model in their general framework. As explained in [7], the model was motivated by material sciences. For instance, it can be used to model the effect of fractures in a material to the overall strength and brittleness of said material, or when studying fault lines in geological structures.

Since the introduction of this model, a host of papers on the subject has appeared in the physics literature (see for example [8] and [10] and the references therein). Many of these deal with a three-dimensional variant where the two-dimensional stick is replaced by other percolation objects, such as nanotubes or nanowires, which are suspended in some other material. These models have been used to model various phenomena such as thin film transistors, flexible microelectronics, microelectromechanical systems, chemical sensors and the construction of transparent electrodes for optoelectronic and photovoltaic devices (as

Communicated by Aernout van Enter.

Supported by the Swedish Research Council.

$凶$ Erik I. Broman

broman@chalmers.se

1 Chalmers University of Technology and Gothenburg University, Göteborg, Sweden 
stated in [8]). In order to have a concrete example in mind, we can consider a material consisting of conductive nanowires suspended in a non-conductive substance. The material is then said to be conductive if large connected components of nanowires exist through which current can flow.

The main purpose and motivation of this paper is to perform a rigorous mathematical analysis of such models in higher-dimensional space. The physically most relevant examples are clearly when $d=2$ (e.g. thin films) or $d=3$ (e.g. conductivity of suspended nanowires), but from a mathematical viewpoint it is desirable to obtain a general result that works for all $d \geq 2$.

A secondary motivation comes from the recent study (see [11] and [1]) of the so-called Poisson cylinder model. This is a model where the percolation objects are infinitely long, and while it was shown in [11] that the vacant set undergoes a phase transition, it was later shown in [1] that the occupied component does not. (We point out that, since the cylinders are unbounded, the phase transition in question is not whether there exists unbounded connected components, but rather whether all cylinders in the model belong to the same connected set.) It is natural to think of the Poisson cylinder model as a limit of the Poisson stick model as the length $L$ of the sticks diverges. From this viewpoint it then becomes natural to ask how the percolation threshold behaves in this limit.

The main model (see Sect. 2.1 for a precise definition) studied in this paper can informally be described as follows. Start with a homogeneous Poisson point process in $\mathbb{R}^{d}$ where $d \geq 2$. For every point $x$ belonging to this process, we place a stick centered at $x$ and we let this stick be of length $L$ and radius 1 . Then, we let the orientation of the sticks be chosen independently and according to some distribution. In this paper we will mainly focus on two cases. Firstly, the uniform case in which the orientation distribution is uniform, and secondly, the rigid case where the orientation is always along the same direction. The reason for considering these two cases is that they represent two extremes among all possible choices of distributions. We point out that, while these two cases are our main focus, our results hold for a more general setting (see Theorem 3.1).

Let $\lambda$ denote the intensity of the Poisson point process. As usual, we say that percolation occurs if the sticks form at least one unbounded connected component. Furthermore, we let $\lambda_{c, u}=\lambda_{c, u}(L)$ denote the critical value of the percolation phase transition (see Sect. 2.1 for a precise definition) in the uniform case, and we let $\lambda_{c, r}=\lambda_{c, r}(L)$ denote the corresponding critical value in the rigid case. The focus of this paper is the behavior of $\lambda_{c, u}(L)$ and $\lambda_{c, r}(L)$ as $L \rightarrow \infty$. As a motivation we informally consider a variant of the uniform case when $d=2$. Consider in this case a model with sticks of length 1 and width 0 , while the intensity is $\lambda$. Then, we increase the length to $L>1$, after which we rescale space by a factor of $L^{-1}$ to recover sticks of length 1 . After these two steps we are back with the original model, but with a new intensity which is $L^{2} \lambda$. From this, one sees that the critical value for this model must scale like $L^{-2}$. Of course, this scaling is exact since the width of the sticks is 0 rather than 1, but morally we should then have that also $\lambda_{c, u}(L)$ scales like $L^{-2}$ when $d=2$. The reason for this is that when $d=2$, in order for two sticks to overlap, the center lines of the sticks tend to cross each other, rather than the sticks just touching. Thus in this case, the width plays a minor role. However, when $d \geq 3$, no center lines will cross and so the width is crucial. We can therefore not generalize the intuition from $d=2$, and it is natural to investigate what happens when $d \geq 3$.

There are two main results of this paper corresponding to the two above mentioned cases. The uniform case will by far require the most effort and so we present this result first. 
Theorem 1.1 For any dimension $d \geq 2$, there exist constants $0<c<C<\infty$, only depending on the dimension, such that for every L large enough,

$$
c L^{-2} \leq \lambda_{c, u}(L) \leq C L^{-2} .
$$

Remarks Let us informally turn to the applications of conductivity of nanowires suspended in some other substance. Then, the implication of Theorem 1.1 is that a doubling of the length of these wires implies that roughly a quarter of the original number of sticks will suffice in order to maintain connectivity. Theorem 1.1 shows that this result does not depend on $d$ other than (possibly) through the values of the constants.

We do not have an easy intuitive argument for why the scaling should be the same in all dimensions. However, it is possible to provide a back-of-the-envelope calculation indicating that the scaling should indeed be $L^{-2}$. This is done in Sect. 3 .

We prove a slightly stronger version of Theorem 1.1, (i.e. Theorem 3.1) in which we provide explicit bounds on the constants $c$ and $C$ of Theorem 1.1. Furthermore, this theorem also provides a lower bound on how large $L$ must be in order for the result to hold. However, since both the values of the constants and the bound for $L$ are presumably far from optimal, we chose not to state them here.

As will be clear from the proofs, the lower bound of Theorem 1.1 is in fact universal for every orientation distribution of the sticks. Furthermore, Theorem 3.1 provides a result which holds for every orientation distribution where the density is uniformly bounded from below.

It is natural to suspect that $L^{-2}$ is not the correct scaling for every orientation distribution, and indeed, the rigid case exhibits a different scaling as stated in our second main result.

Theorem 1.2 For any dimension $d \geq 2$, there exist constants $0<c<C<\infty$, only depending on the dimension, such that for every L large enough we have that

$$
c L^{-1} \leq \lambda_{c, r}(L) \leq C L^{-1} .
$$

Remark As we will see, the proof of Theorem 1.2 will be easier than the proof of Theorem 1.1. Again, we will in be able to provide explicit bounds on the constants $c$ and $C$ (see Theorem 3.2), but as with Theorem 1.1, these are presumably far from optimal. In addition we will provide a lower bound on $L$ such that the statement holds.

One may ask whether any of the two cases considered here are physically realistic, and one might desire a more complete picture. Ideally, one would like to have a result where the scaling can be expressed as a function of the orientation distribution for any such distribution. At this point we do not see a way to prove such a result. However, we do believe that the techniques used in this paper can be used to study also other (special) cases of orientation distributions, but that including more cases would obfuscate the clarity of the arguments and would unnecessarily lengthen the paper.

Even though the results of Theorems 1.1 and 1.2 differ in the sense that they establish different scaling behavior, they in fact rely on the same proof techniques. The proofs of the lower bounds (i.e. $\lambda_{c, u} \geq c L^{-2}$ and $\lambda_{c, r} \geq c L^{-1}$ ) will be completed by coupling the stick percolation model with a subcritical Galton-Watson process (see the beginning of Sect. 4 for a somewhat longer heuristic explanation). The proofs of the upper bounds (i.e. $\lambda_{c, u} \leq C L^{-2}$ and $\lambda_{c, r} \geq C L^{-1}$ ) will be based on an explicit construction of the unbounded components, comparing them to so-called oriented percolation (see the beginning of Sect. 5 for some heuristics).

The rest of this paper is organized as follows. In Sect. 2 we introduce necessary notation and define and explain the models and setup. In Sect. 3 we will state the stronger versions of 
Theorems 1.1 and 1.2. In Sect. 4 we will prove the lower bounds of these two theorems, while the upper bounds will be proven in Sect. 5. The arguments of Sects. 4 and 5 (in particular the latter) will rely on some fairly long calculations. In order not to interrupt the flow of reading more than necessary, these calculations have been put in an appendix (Appendix A).

\section{The Poisson Stick Model}

In this section, we will give formal definitions of the general Poisson stick model and the two orientation distributions considered in this paper. However, we will start by briefly addressing some basic notation.

We will let $\|x\|$ denote the regular $L^{2}$-norm of $x \in \mathbb{R}^{d}$, and for $A, B \subset \mathbb{R}^{d}$ we will write $\operatorname{dist}(A, B):=\inf _{x \in A, y \in B}\|x-y\|$ for the distance between the sets $A, B$. For any set $A \subset \mathbb{R}^{d}$ we let $\operatorname{Vol}(A)$ denote the $d$-dimensional Lebesgue measure of $A$, and we will let

$$
A^{+a}:=\left\{x \in \mathbb{R}^{d}: \operatorname{dist}(x, A) \leq a\right\},
$$

denote an enlargement of $A$.

Throughout, $B(x, r) \subset \mathbb{R}^{d}$ will refer to a (closed) ball centered at $x$ and with radius $r>0$. In a few places we will work in $\mathbb{R}^{d}$ but consider balls in $\mathbb{R}^{d-1}$. In these places we will write $B_{d-1}(x, r)$ in order to emphasize that it is a subset of $\mathbb{R}^{d-1}$. Furthermore, $\operatorname{Vol}\left(B_{d-1}(x, r)\right)$ will then refer to the $(d-1)$-dimensional volume of said ball.

We will let $e_{1}, \ldots, e_{d}$ denote the standard unit directions in $\mathbb{R}^{d}$ while we let $\langle x, y\rangle$ denote the usual scalar product of $x, y \in \mathbb{R}^{d}$. Furthermore, for any $x=\left(x_{1}, x_{2}, \ldots, x_{d}\right) \in \mathbb{R}^{d}$ we will let

$$
x^{r}=\left(r, x_{2}, \ldots, x_{d}\right) .
$$

Throughout, we will use $o \in \mathbb{R}^{d}$ and $o \in \mathbb{Z}^{d}$ to denote the origin.

\subsection{The General Poisson Stick Model}

We start by considering the space $\mathbb{R}^{d} \times \mathbb{S}$ where $\mathbb{S}=\left\{p \in \mathbb{R}^{d}:\|p\|=1\right\}$ is the unit sphere in $\mathbb{R}^{d}$. Then, for any pair $(x, p) \in \mathbb{R}^{d} \times \mathbb{S}$ we associate the line segment

$$
\ell_{x, p, L}=\{x+t p:-L / 2 \leq t \leq L / 2\} .
$$

This is a line segment of length $L$ with orientation vector $p$ centered at $x$. Then, we define

$$
S_{x, p, L}=\left\{y \in \mathbb{R}^{d}: \operatorname{dist}\left(y, \ell_{x, p, L}\right) \leq 1\right\},
$$

so that $S_{x, p, L}=\ell_{x, p, L}^{+1}$. We will refer to $S_{x, p, L}$ as a stick. Note that the "tips" of the sticks are rounded, and so the sticks are not truncated cylinders. This is a matter of convenience and will have no qualitative effect on the results of this paper (although it may affect the constants involved in the bounds of Theorems 1.1 and 1.2). Indeed, one can easily sandwich a stick in between two truncated cylinders of lengths $L$ and $L+2$ respectively. Clearly, $S_{x, p, L}=S_{x,-p, L}$, but this will not be an issue.

Next we define the intensity measure $\mu_{\lambda}$ on $\mathbb{R}^{d} \times \mathbb{S}$ which we will use for our Poisson point process. Let

$$
\mu_{\lambda}(\mathrm{d} x, \mathrm{~d} p)=\lambda \operatorname{Leb}(\mathrm{d} x) \otimes \Theta(\mathrm{d} p)
$$


where $\lambda>0$ is a parameter, $\operatorname{Leb}(\mathrm{d} x)$ denotes Lebesgue measure on $\mathbb{R}^{d}$ and $\Theta(\mathrm{d} p)$ denotes a probability distribution on $\mathbb{S}$. Next, let

$$
\Omega=\left\{\omega \subset \mathbb{R}^{d} \times \mathbb{S}:|\omega \cap(A \times \mathbb{S})|<\infty \text { whenever } \operatorname{Leb}(A)<\infty\right\},
$$

be the space of configurations. We will let $\Pi^{\lambda}$ denote a Poisson point process on $\mathbb{R}^{d} \times \mathbb{S}$ with intensity measure $\mu_{\lambda}$. Since $\Theta(\mathbb{S})=1$, it follows that $\Pi^{\lambda}$ is a random element of $\Omega$. We will write $(x, p) \in \Pi^{\lambda}$ for a point in $\Pi^{\lambda}$. Clearly, $\Pi^{\lambda}$ induces a Poisson point process of sticks in $\mathbb{R}^{d}$ by identifying a point $(x, p)$ with the stick $S_{x, p, L}$. Consider then

$$
\mathcal{C}\left(\Pi^{\lambda}\right)=\bigcup_{(x, p) \in \Pi^{\lambda}} S_{x, p, L},
$$

which is referred to as the occupied set. We say that percolation occurs if $\mathcal{C}\left(\Pi^{\lambda}\right)$ contains a connected unbounded component. Following Sect. 2.1 in [7], one can use ergodicity to prove that percolation is a $0-1$ event. It is therefore natural to define the critical threshold $\lambda_{c, \Theta}=\lambda_{c, \Theta}(L)$ for which percolation occurs by letting

$$
\lambda_{c, \Theta}:=\inf \left\{\lambda>0: \mathbb{P}\left(\mathcal{C}\left(\Pi^{\lambda}\right) \text { percolates }\right)=1\right\} .
$$

\subsection{The Uniform and the Rigid Cases}

Let $\mathcal{H}(\mathrm{d} p)$ denote the $(d-1)$-dimensional normalized Hausdorff measure on $\mathbb{S}$ so that

$$
\mathcal{H}(\mathbb{S})=\int_{\mathbb{S}} \mathcal{H}(\mathrm{d} p)=1
$$

Clearly, $\mathcal{H}(\mathrm{d} p)$ corresponds to uniform distribution of the sticks, and in this case we let $\lambda_{c, u}$ denote the quantity defined by (2.5).

The second case we consider in this paper is when all sticks are oriented in the same direction. Clearly, the choice of direction is not important and so for definiteness we will consider $\Theta(\mathrm{d} p)=\delta_{e_{2}}$. Here, we let $\lambda_{c, r}$ denote the quantity corresponding to (2.5).

\section{Alternative Statements}

We will now state the stronger versions of Theorems 1.1 and 1.2 mentioned in the introduction.

The statement of Theorem 3.1 (which is the stronger version of Theorem 1.1) contains a lower and an upper bound. The lower bound holds for any orientation distribution while the upper bound holds for any orientation distribution $\Theta(\mathrm{d} p)$ such that $\Theta(\mathrm{d} p)=\phi(p) \mathcal{H}(\mathrm{d} p)$ where for some $\delta>0$,

$$
\phi(p) \geq \delta \mathcal{H} \text {-almost surely. }
$$

Since $\Theta(\mathrm{d} p)$ is a probability distribution we clearly have that $\int_{\mathbb{S}} \phi(p) \mathcal{H}(\mathrm{d} p)=1$.

Theorem 3.1 For any $d \geq 2$ and any orientation distribution $\Theta(\mathrm{d} p)$ we have that

$$
\lambda_{c, \Theta} \geq \frac{\Gamma((d+1) / 2)}{\pi^{(d-1) / 2} 2^{d}} L^{-2}
$$


for any $L>\pi$. Furthermore, for any $d \geq 2$ and any orientation distribution such that $\Theta(\mathrm{d} p)=\phi(p) \mathcal{H}(\mathrm{d} p)$ where $\phi(p)$ satisfies (3.1) for some $\delta>0$, we have that

$$
\lambda_{c, \Theta} \leq \frac{20(1000 \sqrt{d})^{d} \sqrt{d} \Gamma(2 d-1)}{9 \delta 2^{5(d-2)} \pi^{d / 2-2} \Gamma(d / 2)^{3}} L^{-2}
$$

for $L>200 \sqrt{d}$.

Remark The requirement that $L>200 \sqrt{d}$ can be improved. However, this would mean more technical details in the proofs, and since we are interested in the asymptotics, this does not seem worthwhile.

Proof of Theorem 1.1 from Theorem 3.1 This is a trivial consequence of Theorem 3.1 by considering the case when $\phi(p) \equiv 1$.

Before turning to our second result, we will provide the back-of-the-envelope calculation showing why $L^{-2}$ should be the correct scaling in Theorem 1.1. We will be very informal.

Step 1: Start by considering an "almost-horizontal" stick. Since that stick is of length $L$, there should be order $L$ independent segments where an "almost-vertical" stick can hit it.

Step 2: Most almost-vertical sticks that are within range to hit the first stick, have their centers at distance order $L$ from the first stick. Ignoring all other sticks, such a stick must by spherical symmetry have probability of order $L^{-d+1}$ to hit one of the independent segments on the first stick.

Step 3: The volume at which the center of the almost-vertical stick can be located and still hit the almost-horizontal stick must be of order $L^{d}$. The constant in front of $L^{d}$ will be small. How small depends on the exact definition of almost-vertical.

Step 4: We see that the expected number of almost-vertical sticks hitting the almosthorizontal is then of order $\lambda \cdot L \cdot L^{-d+1} \cdot L^{d}=\lambda L^{2}$. Thus, if $\lambda$ equals a large constant times $L^{-2}$ we should have a good chance of finding a pair of sticks forming an $L$-shaped figure. With this basic building block we should be able to construct an unbounded component. From this we deduce that the correct scaling of $\lambda_{c, u}(L)$ must be $L^{-2}$.

There are of course several issues with the above calculations. For instance, we are ignoring everything which is not almost-horizontal or almost-vertical. Perhaps this is giving up too much? In addition, we ignore almost-vertical sticks whose centers are closer to the almosthorizontal than order $L$. Perhaps these are essential since they have a better chance of hitting the almost-horizontal stick? The answers to both of these questions are (in light of the results of this paper) no, but clearly some care will be needed in order to provide a rigorous proof.

We now turn to the stronger version of Theorem 1.2 that includes explicit values of the constants involved. Recall that the rigid model is when all sticks are oriented along the same direction, and that we chose $\Theta(\mathrm{d} p)=\delta_{e_{2}}$ for definiteness.

Theorem 3.2 For any dimension $d \geq 2$, and for every $L>3$, we have that

$$
\lambda_{c, r}(L) \geq \frac{\Gamma((d+1) / 2)}{2^{d} \pi^{d / 2}} L^{-1} .
$$

Furthermore, for every $L>10$, we have that

$$
\lambda_{c, r}(L) \leq 4 \frac{2^{d} \Gamma((d+1) / 2)}{\pi^{d / 2-1}} L^{-1} .
$$


Remark We see by comparing the lower bounds of Theorems 3.1 and 3.2, that even though the lower bound of Theorem 3.1 holds for any orientation distribution, it does not mean that it is always a good bound.

We will consider the upper and lower bounds in Theorems 3.1 and 3.2 in separate sections. It makes sense to group the bounds in the same direction together, as the proof techniques are similar.

We end this section with a short discussion on the bounds provided by Theorems 3.1 and 3.2. Considering that the constants in the upper bounds and the lower bounds of these theorems differ so greatly, one realizes that at least some of them (and maybe all of them) must be far from optimal. Considering the somewhat complicated expressions of the involved constants, one may also question the value of providing these explicit expressions at all. The reason for doing so is in part that it makes it easier to follow the proofs, since we never have to write things like "for $c$ small enough" or "for $L$ large enough" (except when providing heuristics). It also makes it easier for the reader to verify just how poor the bounds are. For instance, one could compare these to numerical bounds that may be provided in the future.

\section{The Lower Bounds}

The lower bounds of Theorems 3.1 and 3.2 will be proven along the same lines. We will begin this section by giving an informal explanation of the involved argument.

To that end, consider a single stick placed at the origin (the orientation will not be important here). Then, explore the Poisson point process in order to find any sticks intersecting this first stick. The next step in turn then consists of exploring those sticks found in the previous step, and so on. This stick exploration procedure will be coupled to a Galton-Watson process. Elementary measure calculations (which can be found in Appendix A.1) will show that this Galton-Watson process is subcritical whenever $\lambda<c L^{-2}$ and $c=c(d)>0$ small enough in the uniform case, while it is subcritical whenever $\lambda<c L^{-1}$ and $c=c(d)>0$ small enough in the rigid case. For such $\lambda$, we can conclude that the component of the original stick at the origin will be finite almost surely, and the lower bounds will follow.

We now turn to the proof of the lower bound of Theorem 3.1. This will require us to know the measure of the set of line segments that hit a ball of radius 2 . However, we will prove the statement for any radius $\rho>0$ as this can be done with no extra effort. The proof of this result (Lemma 4.1) is postponed until Appendix A.1.

Lemma 4.1 (Measure of line segments hitting a ball) For any $d \geq 2, L, \rho>0$ and any distribution $\Theta(\mathrm{d} p)$ we have that

$$
\begin{aligned}
& \mu_{\lambda}\left((x, p) \in \mathbb{R}^{d} \times \mathbb{S}: \ell_{x, p, L} \cap B(o, \rho) \neq \emptyset\right) \\
& \quad=\operatorname{Vol}\left(S_{o, p, L}(\rho)\right)=L \frac{\pi^{(d-1) / 2}}{\Gamma((d+1) / 2)} \rho^{d-1}+\frac{\pi^{d / 2}}{\Gamma(d / 2+1)} \rho^{d} .
\end{aligned}
$$

Equipped with this lemma we can now prove the lower bound of Theorem 3.1. Throughout most of this section, the location of the center point $x$ and the direction $p$ of a line segment $\ell_{x, p, L}$ will not explicitly be used. It is therefore convenient to abuse notation and simply write $\ell \in \Pi^{\lambda}$ for a line segment corresponding to some point in the Poisson point process, and $S_{\ell}$ for the corresponding stick. For $A \subset \mathbb{R}^{d}$, we will also write $\mu_{\lambda}\left(\ell: S_{l} \cap A \neq \emptyset\right)$ instead of $\mu_{\lambda}\left((x, p) \in \mathbb{R}^{d} \times \mathbb{S}: S_{x, p, L} \cap A \neq \emptyset\right)$. 
Proof of lower bound of Theorem 3.1 Although the idea behind the proof is fairly straightforward, some care is needed when constructing the actual coupling. In order to explain the idea, fix some line segment $\ell_{0}$, let $\Pi^{\lambda}$ be as in Sect. 2.1, and let $\left(\Pi_{k, n}^{\lambda}\right)_{k, n \geq 1}$ be an i.i.d. collection of random variables which are independent copies of $\Pi^{\lambda}$. Then, we let $\mathcal{C}_{0}\left(\Pi^{\lambda}\right)$ denote the connected component (if it exists) of $\mathcal{C}\left(\Pi^{\lambda}\right)$ such that $S_{\ell_{0}} \cap \mathcal{C}_{0}\left(\Pi^{\lambda}\right) \neq \emptyset$. The aim is to prove that $\mathcal{C}_{0}\left(\Pi^{\lambda}\right)$ is a bounded set almost surely whenever $\lambda<c L^{-2}$ where $c=c(d)$ is small enough. We will prove this by stochastically comparing $\mathcal{C}_{0}\left(\Pi^{\lambda}\right)$ to something larger, but which is easier for us to analyze. By our construction, this larger object will naturally correspond to a subcritical branching process. We can then conclude that this larger object must be bounded, and from this it will immediately follow that $\mathcal{C}\left(\Pi^{\lambda}\right)$ cannot contain any unbounded connected components.

We start by letting

$$
\Psi_{1}^{\lambda}=\left\{\ell \in \Pi^{\lambda}: S_{\ell} \cap S_{\ell_{0}} \neq \emptyset\right\},
$$

so that $\Psi_{1}^{\lambda}$ consists of those line segments in $\Pi^{\lambda}$ whose corresponding sticks intersect $S_{\ell_{0}}$. Let $\ell_{1,1}, \ldots, \ell_{\left|\Psi_{\lambda}^{1}\right|, 1}$ be an enumeration of the line segments in $\Psi_{\lambda}^{1}$, and we think of these as the line segments of generation 1.

Next, we let

$$
\Psi_{1,2}^{\lambda}=\left\{\ell \in \Pi^{\lambda} \backslash \Psi_{1}^{\lambda}: S_{\ell} \cap S_{\ell_{1,1}} \neq \emptyset\right\} \cup\left\{\ell \in \Pi_{1,1}^{\lambda}: S_{\ell} \cap S_{\ell_{1,1}} \neq \emptyset, S_{\ell} \cap S_{\ell_{0}} \neq \emptyset\right\} .
$$

The first set of line segments in this union are the segments $\ell \in \Pi^{\lambda}$ such that $S_{\ell} \cap S_{\ell_{1,1}} \neq \emptyset$, but that we did not already encounter when defining $\Psi_{1}^{\lambda}$ (i.e. they did not intersect $S_{\ell_{0}}$ ). The second set of line segments are then "extra" segments from $\Pi_{1,1}^{\lambda}$ which are required to hit both $S_{\ell_{1,1}}$ and $S_{\ell_{0}}$. The reason for adding these extra line segments is that now, $\Psi_{1}^{\lambda}$ and $\Psi_{1,2}^{\lambda}$ are both generated by considering a Poisson point process of line segments intersecting $S_{\ell_{0}}^{+1}$ and $S_{\ell_{1,1}}^{+1}$ (recall (2.1)) respectively. Therefore, $\left|\Psi_{1}^{\lambda}\right|$ and $\left|\Psi_{1,2}^{\lambda}\right|$ are equal in distribution, and furthermore, any $\ell \in \Pi^{\lambda}$ such that $S_{\ell}$ intersects $S_{\ell_{0}}$ or $S_{\ell_{1,1}}$ must by construction also belong to $\Psi_{1}^{\lambda} \cup \Psi_{1,2}^{\lambda}$. For $k=2, \ldots,\left|\Psi_{1}^{\lambda}\right|$ we then let

$$
\begin{aligned}
& \Psi_{k, 2}^{\lambda}=\left\{\ell \in \Pi^{\lambda} \backslash\left(\Psi_{1}^{\lambda} \cup_{j=1}^{k-1} \Psi_{j, 2}^{\lambda}\right): S_{\ell} \cap S_{\ell_{k, 1}} \neq \emptyset\right\} \\
& \cup\left\{\ell \in \Pi_{k, 1}^{\lambda}: S_{\ell} \cap S_{\ell_{k, 1}} \neq \emptyset, S_{\ell} \cap\left(S_{\ell_{0}} \cup_{j=1}^{k-1} S_{\ell_{j, 1}}\right) \neq \emptyset\right\} .
\end{aligned}
$$

As above, the first set is the set of line segments in $\Pi^{\lambda}$ not yet encountered. We think of this as using $\Pi^{\lambda}$ wherever $\Pi^{\lambda}$ has not already been explored/used. The second part is then an "extra" set of line segments, i.e. we use $\Pi_{k, 1}^{\lambda}$ wherever $\Pi^{\lambda}$ has already been used. By adding these segments, we compensate for the space already explored, and so $\left|\Psi_{k, 2}^{\lambda}\right|$ where $k=1,2, \ldots,\left|\Psi_{1}^{\lambda}\right|$ becomes an i.i.d. sequence of random variables, all with the same distribution as $\left|\Psi_{1}^{\lambda}\right|$. Next, we let

$$
\Psi_{2}^{\lambda}=\bigcup_{k=1}^{\left|\Psi_{1}^{\lambda}\right|} \Psi_{k, 2}^{\lambda},
$$

and note that if $\ell \in \Pi^{\lambda}$ is such that we can jump from $S_{\ell_{0}}$ to $S_{\ell}$ by using at most one other stick in $\Pi^{\lambda}$, our construction gives us that $\ell \in \Psi_{1}^{\lambda} \cup \Psi_{2}^{\lambda}$. We then enumerate the line segments $\ell_{1,2}, \ldots, \ell_{\mid \Psi_{2}^{\lambda \mid, 2}}$ in $\Psi_{\lambda}^{2}$, and think of these as the segments of generation 2 . 
The general step is performed in the same way. We define the collection $\left(\Psi_{k, n+1}^{\lambda}\right)_{1 \leq k \leq\left|\Psi_{n}^{\lambda}\right|}$ and let

$$
\Psi_{n+1}^{\lambda}=\bigcup_{k=1}^{\left|\Psi_{n}^{\lambda}\right|} \Psi_{k, n+1}^{\lambda} \text { and finally } \Psi^{\lambda}=\bigcup_{n=1}^{\infty} \Psi_{n}^{\lambda} .
$$

If we let $\mathcal{C}_{0}\left(\Psi^{\lambda}\right)=S_{\ell_{0}} \bigcup_{\ell \in \Psi^{\lambda}} S_{\ell}$ (which by definition is a connected component) we see from our construction that

$$
\mathcal{C}_{0}\left(\Pi^{\lambda}\right) \subset \mathcal{C}_{0}\left(\Psi^{\lambda}\right)
$$

It is not hard to see that the sequence $\left(\left|\Psi_{n}^{\lambda}\right|\right)_{n=1}^{\infty}$ corresponds to the sizes of the generations of a Galton-Watson family tree. In order to show that this is subcritical for small enough values of $\lambda$, we must now estimate $\mathbb{E}\left[\left|\Psi_{1}^{\lambda}\right|\right]$. To that end, note that since the length of $\ell$ is $L$, we can cover any stick $S_{\ell}$ by using at most $L$ balls of radius 2 whenever $L \geq 1$ (which holds by assumption). If $S$ is such a stick and $z_{1}, \ldots, z_{L}$ denote the centers of the balls in such a covering, we see that for some $C<\infty$,

$$
\begin{aligned}
& \mu_{\lambda}\left(\ell: S_{\ell} \cap S \neq \emptyset\right) \\
& \leq \bigcup_{k=1}^{L} \mu_{\lambda}\left(\ell: \ell \cap B\left(z_{k}, 2\right) \neq \emptyset\right)=L \mu_{\lambda}\left(\ell: \ell \cap B\left(z_{1}, 2\right) \neq \emptyset\right) \\
& =L \lambda\left(L \frac{\pi^{(d-1) / 2}}{\Gamma((d+1) / 2)} 2^{d-1}+\frac{\pi^{d / 2}}{\Gamma(d / 2+1)} 2^{d}\right) \leq \lambda L^{2} \frac{\pi^{(d-1) / 2}}{\Gamma((d+1) / 2)} 2^{d}
\end{aligned}
$$

by using Lemma 4.1 with $\rho=2$ and where the last inequality holds for any

$$
L \geq 2 \sqrt{\pi} \frac{\Gamma((d+1) / 2)}{\Gamma(d / 2+1)} .
$$

By using that the gamma function is a logarithmically convex function, it is easy to show that the right-hand side of (4.3) is decreasing in $d \geq 2$. Furthermore, the expression equals $\pi$ for $d=2$ and since we assume that $L>\pi$ we conclude that (4.3) holds, and in turn that (4.2) holds. Using this, we then see that

$$
\mathbb{E}\left[\left|\Psi_{1}^{\lambda}\right|\right]=\mu_{\lambda}\left(\ell: S_{\ell} \cap S \neq \emptyset\right) \leq \lambda L^{2} \frac{\pi^{(d-1) / 2}}{\Gamma((d+1) / 2)} 2^{d}
$$

which is strictly smaller than one whenever

$$
\lambda<\frac{\Gamma((d+1) / 2)}{\pi^{(d-1) / 2} 2^{d}} L^{-2} .
$$

For such values of $\lambda$, the corresponding branching process is subcritical and therefore it dies out almost surely. Then, we conclude that $\left|\Psi^{\lambda}\right|<\infty$ almost surely, and so by (4.1) we must also have that $\mathcal{C}_{0}\left(\Pi^{\lambda}\right)$ is bounded almost surely.

Since the choice of $S_{\ell_{0}}$ was arbitrary, it follows by standard Poisson point process theory that $\mathcal{C}\left(\Pi^{\lambda}\right)$ cannot contain any unbounded connected components, and so we conclude that

$$
\lambda_{c, \Theta} \geq \frac{\Gamma((d+1) / 2)}{\pi^{(d-1) / 2} 2^{d}} L^{-2} .
$$

Next we turn to the rigid case. 
Proof of lower bound of Theorem 3.2 The proof is very similar to the proof of the lower bound of Theorem 3.1; the only difference lies in the estimate of $\mu_{\lambda}\left(\ell: S_{\ell} \cap S \neq \emptyset\right)$ in (4.4). In order to estimate this, consider a stick $S_{o, e_{1}, L}$. If $S_{x, e_{1}, L} \cap S_{o, e_{1}, L} \neq \emptyset$, it is necessary (but not sufficient) that $x_{1} \in[-L-2, L+2]$ and that $\left\|x^{0}\right\|<2$ (recall (2.2)). Note that the reason that it is not $x_{1} \in[-L, L]$ is due to the "tips" of the sticks. We therefore see that (recall the notation $B_{d-1}(x, r)$ from Sect. 2)

$$
\begin{aligned}
& \mathbb{E}\left[\left|\Psi_{1}^{\lambda}\right|\right]=\mu_{\lambda}\left(\left(x, e_{1}\right): S_{x, e_{1}, L} \cap S_{o, e_{1}, L} \neq \emptyset\right) \\
& \quad \leq \lambda(2 L+4) \operatorname{Vol}\left(B_{d-1}(o, 2)\right)=\lambda(2 L+4) \frac{\pi^{(d-1) / 2}}{\Gamma((d-1) / 2+1)} 2^{d-1} \\
& \quad \leq \lambda 2^{d} \frac{\pi^{d / 2}}{\Gamma((d+1) / 2)} L,
\end{aligned}
$$

where the last inequality holds for $L>3$. Replacing the estimate in (4.4) with this expression gives us the result.

\section{The Upper Bounds}

In this subsection we will provide proofs of the upper bounds of Theorems 3.1 and 3.2. Here, we will couple the stick process with a so-called oriented percolation model. We will attempt to construct an unbounded component, and the coupling will then show that this construction has positive probability of succeeding when $\lambda>C L^{-2}$ (with a suitable choice of $C=C(d)<\infty$ ) for the uniform case. A similar construction and coupling will work analogously whenever $\lambda>C L^{-1}$ (again with a suitable choice of $C=C(d)<\infty$ ) in the rigid case. This construction will require measure calculations which are mainly postponed until Appendix A.2.

Since both proofs are done through a comparison with the oriented percolation model (although using slightly different variants in the two cases), we will start by introducing said model. To that end, consider the following lattice in the upper half-plane,

$$
\mathbb{H}:=\left\{u \in \mathbb{Z}^{2}: u_{1}+u_{2} \text { is even, and } u_{2} \geq 0\right\} .
$$

We will consider a collection $\left(A_{n}\right)_{n \geq 0}$ of random subsets of $\mathbb{H}$ where $A_{0}=\{o\}$ and where $A_{n}$ is such that

$$
A_{n} \subset\left\{u \in \mathbb{H}: u_{2}=n\right\} .
$$

Given $A_{n}$, the events $\left\{u \in A_{n+1}\right\}$ are conditionally independent with

$$
\mathbb{P}\left(u \in A_{n+1} \mid A_{n}\right)= \begin{cases}\beta & \text { if }\left|A_{n} \cap\{u+(-1,-1), u+(1,-1)\}\right|=2 \\ \alpha & \text { if }\left|A_{n} \cap\{u+(-1,-1), u+(1,-1)\}\right|=1 \\ 0 & \text { if }\left|A_{n} \cap\{u+(-1,-1), u+(1,-1)\}\right|=0\end{cases}
$$

Here, $0<\alpha, \beta<1$, and we will consider two variants. In the first, $\beta=1-(1-\alpha)^{2}$ and in the second, $\beta=\alpha$.

We will give an informal description of this model and the two variants. Consider the first variant where $\beta=1-(1-\alpha)^{2}$. With $A_{0}=\{o\}$, we obtain from (5.2) that for $u=(-1,1)$ and $u=(1,1)$ we have that

$$
\mathbb{P}\left(u \in A_{1}\right)=\alpha
$$


independently for $u=(-1,1)$ and $u=(1,1)$. We can think of this as drawing an arrow with probability $\alpha$ from $o$ to $(-1,1)$ and independently, with the same probability, drawing one from $o$ to $(1,1)$. In general, conditional on $A_{n}$, then if $u \in A_{n}$, we draw an arrow with probability $\alpha$ from $u$ to $u+(1,1)$, and independently (again with probability $\alpha$ ), from $u$ to $u+(-1,1)$. This is done independently for every $u \in A_{n}$. Then, every $v \in \mathbb{H}$ which is pointed at by an arrow emanating from some $u \in A_{n}$ belongs to $A_{n+1}$. Clearly, this is a bond percolation model. The second variant (i.e. when $\beta=\alpha$ ) is in contrast a site percolation model since here $\mathbb{P}\left(u \in A_{n+1} \mid A_{n}\right)=\alpha$ whether there are one or two sites in $A_{n}$ "preceeding" $u$.

The bond version of this model was introduced in [3] where it is proven that if $\alpha<1$ is large enough,

$$
\mathbb{P}\left(A_{n} \neq \varnothing \quad \forall n \geq 0\right)>0 .
$$

In order for us to obtain the explicit bounds required in Theorems 3.1 and 3.2, we shall need some explicit bounds on $\alpha$ such that (5.3) holds, and we require this for both variants. It was proven in [6] that for the bond version, any $\alpha>2 / 3$ is such that (5.3) holds, while for the site version, (5.3) holds whenever $\alpha>3 / 4$.

We will use the bond model to prove the upper bound of Theorem 3.1, although we do this on the lattice $2 \mathbb{H}$ (this is a matter of notational convenience and clearly does not matter). When proving the upper bound of Theorem 3.2, we instead use the site model on $\mathbb{H}$.

\subsection{The Upper Bound of Theorem 3.1}

We start this subsection by providing the intuition along with some necessary notation. The proof will rely on a construction of an unbounded component which essentially will be performed on a two-dimensional lattice. To that end, for any $u \in \mathbb{Z}^{2}$ we will write

$$
D^{u}=\left(u_{1}, u_{2}, 0, \ldots, 0\right) \frac{L}{4}+\left[-\frac{L}{16 \sqrt{d}}, \frac{L}{16 \sqrt{d}}\right]^{d} \text {, }
$$

where $\left(u_{1}, u_{2}, 0, \ldots, 0\right) \in \mathbb{Z}^{d}$. Note that $D^{u} \cap D^{v}=\emptyset$ whenever $u \neq v$. We then start by considering the three boxes $D^{(-2,0)}, D^{(-1,0)}$ and $D^{o}$. For any $\lambda>0$ there is a positive probability that there exists $(x, p) \in \Pi^{\lambda}$ with the following three properties. Firstly, the center of $\ell_{x, p, L}$ (i.e. $x$ ) belongs to $D^{(-1,0)}$. Secondly, $\ell_{x, p, L}$ intersects $D^{(-2,0)}$, and lastly $\ell_{x, p, L}$ intersects the "right-hand" boundary of $D^{o}$. The corresponding stick $S_{x, p, L}$ will then present a target for a second stick (see Fig. 1a for an illustration). This second stick $S_{y, q, L}$ will be required to have its center in the box $D^{(0,1)}$, to hit the target presented by $S_{x, p, L}$ and to intersect the "top" boundary of $D^{(0,2)}$ (see Fig. 1b). This in turn then becomes a target for two additional sticks centered in $D^{(-1,2)}$ and $D^{(1,2)}$, presenting targets in $D^{(-2,2)}$ and $D^{(2,2)}$ respectively. This procedure can be continued and coupled with the oriented percolation model described above.

Our next step is to introduce the following notation which will make the notion of "righthand", "left-hand" and "top" part of the boundary of the boxes $D^{u}$ precise. For any $u \in \mathbb{Z}^{2}$, 


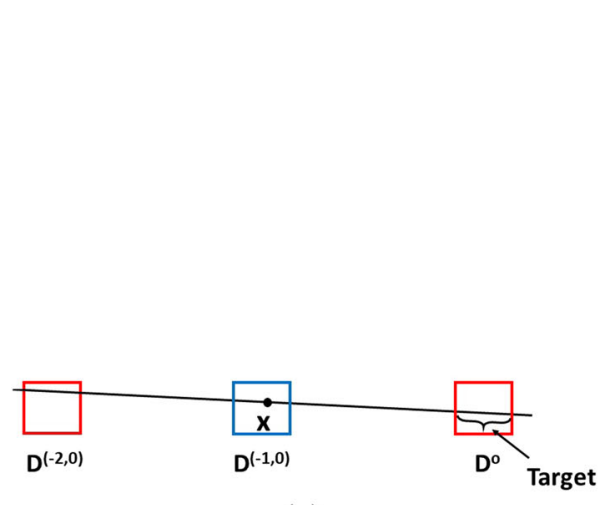

(a)

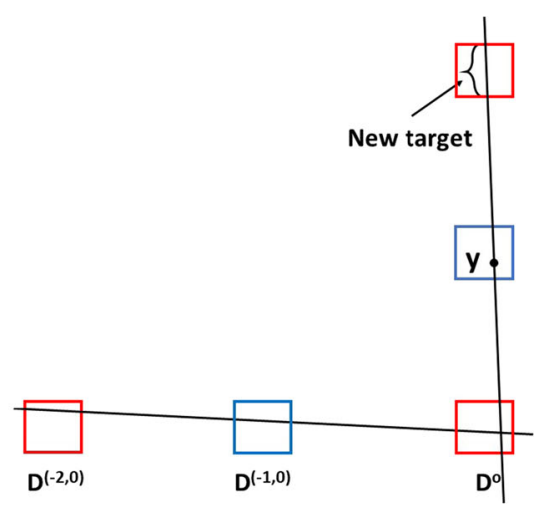

(b)

Fig. 1 In a the stick $S_{x, p, L}$ is centered in $D^{(-1,0)}$, intersects $D^{(-2,0)}$ and "exits" the right-hand side of $D^{o}$. It presents a target for the next step. In $\mathbf{b}$ the stick $S_{y, p, L}$ hits the previous target and presents a new one. Here, blue boxes are those where centers of our sticks will be found, while red boxes are where intersections occur

let

$$
\begin{aligned}
L^{-16}\left(D^{u}\right)= & \left\{x \in D^{u}: x_{1}=u_{1} \frac{L}{4}-\frac{L}{16 \sqrt{d}},\left|x_{2}-u_{2} \frac{L}{4}\right| \leq \frac{L}{16 \sqrt{d}}-16\right. \\
& \text { and } \left.\left|x_{k}\right| \leq \frac{L}{16 \sqrt{d}}-16 \text { for } k=3, \ldots, d\right\}, \\
R^{-16}\left(D^{u}\right)= & \left\{x \in D^{u}: x_{1}=u_{1} \frac{L}{4}+\frac{L}{16 \sqrt{d}},\left|x_{2}-u_{2} \frac{L}{4}\right| \leq \frac{L}{16 \sqrt{d}}-16\right. \\
& \text { and } \left.\left|x_{k}\right| \leq \frac{L}{16 \sqrt{d}}-16 \text { for } k=3, \ldots, d\right\},
\end{aligned}
$$

and

$$
\begin{aligned}
T^{-16}\left(D^{u}\right)= & \left\{x \in D^{u}: x_{2}=u_{2} \frac{L}{4}+\frac{L}{16 \sqrt{d}},\left|x_{1}-u_{1} \frac{L}{4}\right| \leq \frac{L}{16 \sqrt{d}}-16\right. \\
& \text { and } \left.\left|x_{k}\right| \leq \frac{L}{16 \sqrt{d}}-16 \text { for } k=3, \ldots, d\right\}
\end{aligned}
$$

We see that $L^{-16}\left(D^{u}\right), R^{-16}\left(D^{u}\right)$ and $T^{-16}\left(D^{u}\right)$ are subsets of the left-hand, right-hand and top part of the boundary of $D^{u}$ (see also Fig. 2). We will require the line segments of our construction to hit these sets (rather than anywhere on the corresponding faces of $D^{u}$ ). The reason for this is that unless the line segments hit "well inside" the faces, then they will not behave in a way that makes a continuation of the construction possible. We note that we will not need a notation for the bottom part.

For two sets $A, B \subset \mathbb{R}^{d}$ we will write

$$
A \stackrel{\ell_{x, p, L}}{\longrightarrow} B
$$

for the event $\left\{\ell_{x, p, L} \cap A \neq \emptyset\right\} \cap\left\{\ell_{x, p, L} \cap B \neq \emptyset\right\}$. Thus, $B(\gamma, \rho) \stackrel{\ell_{x, p, L}}{\longrightarrow} B(\zeta, \rho)$ is the event that the line segment $\ell_{x, p, L}$ connects the two balls $B(\gamma, \rho)$ and $B(\zeta, \rho)$. Let $\gamma \in D^{(-2,0)}$, 
Fig. 2 We see a box $D^{u}$ centered at $\left(u_{1}, u_{2}, 0\right) L / 4$ and with side length $\frac{L}{8 \sqrt{3}}$. We can also see $R^{-16}\left(D^{u}\right)$ as the solid black area on the right-hand side of the box

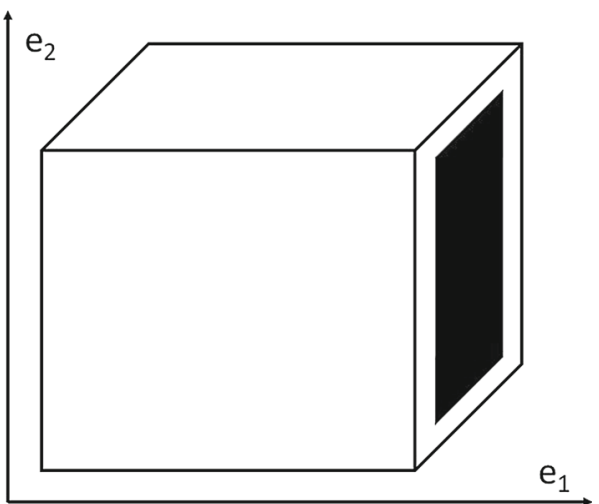

be such that $B(\gamma, 2) \subset D^{(-2,0)}$ and let $\zeta \in D^{o}$. Note that

$$
\begin{aligned}
& \left\{(x, p): x \in D^{(-1,0)}, B(\gamma, 2) \stackrel{\ell_{x, p, L}}{\longleftrightarrow} B(\zeta, 2)\right\} \\
& \quad=\left\{(x, p): x \in D^{(-1,0)}, B(\gamma, 1) \cap S_{x, p, L} \neq \emptyset, B(\zeta, 1) \cap S_{x, p, L} \neq \emptyset\right\},
\end{aligned}
$$

since the ball $B(\gamma, 1)$ and the stick $S_{x, p, L}$ touches if and only if $\ell_{x, p, L}$ comes within distance 2 from $\gamma$. (This is where our choice of definition of a stick with rounded tips, i.e. (2.3), becomes convenient.)

Our next lemma is proved through a number of intermediate steps, and we postpone the proofs of these and the lemma itself until Appendix A.2.

Lemma 5.1 (Measure of line segments connecting two balls) Let $\Theta(\mathrm{d} p)=\phi(p) \mathcal{H}(\mathrm{d} p)$ where $\phi(p)$ satisfies (3.1) for some $\delta>0$. For every $d \geq 2, L>32$ and any $\gamma \in D^{(-2,0)}$, $\zeta \in R^{-16}\left(D^{o}\right)$ we have that

$$
\mu_{\lambda}\left((x, p): x \in D^{(-1,0)}, B(\gamma, 2) \stackrel{\ell_{x, p, L}}{\longleftrightarrow} B(\zeta, 2)\right) \geq \lambda \delta c_{d} L^{-d+2},
$$

where we may take

$$
c_{d}=\frac{2^{5(d-2)} \pi^{d / 2-2}}{\sqrt{d}} \frac{\Gamma(d / 2)^{3}}{\Gamma(2 d-1)} .
$$

Lemma 5.1 will be used to estimate the probability of hitting a target and simultaneously providing a new one (as discussed at the start of this subsection). However, before we are ready to obtain such an estimate, we need to make precise what these notions mean. Recall therefore (2.1) and let $D^{u,+2}:=\left(D^{u}\right)^{+2}$ be an enlargement of $D^{u}$. Note that $D^{u,+2}$ is not the same as $D^{u+(2,2)}$, since the latter refers to the box defined by (5.4) with $u=\left(u_{1}, u_{2}\right)$ so that $u+(2,2)=\left(u_{1}+2, u_{2}+2\right)$. Note also that while $R^{-16}\left(D^{o}\right)$ denotes a "shrunk" version of the right-hand boundary of $D^{o}$ (and therefore of a $(d-1)$-dimensional object), $A^{+a}$ as defined in (2.1) denotes an enlargement in all dimensions.

Fix some $\ell_{x, p, L}$ such that

$$
D^{(-2,0),+2} \stackrel{\ell_{x, p, L}}{\longleftrightarrow} R^{-16}\left(D^{o}\right) .
$$

Given this $\ell_{x, p, L}$, let

$$
\mathcal{T}\left(\ell_{x, p, L}, D^{o}\right)=\left\{y \in \mathbb{R}^{d}: \operatorname{dist}\left(y, \ell_{x, p, L} \cap D^{o}\right) \leq 1\right\},
$$


which we think of as the (horizontal) target presented by $S_{x, p, L}$ for the next step. Note that $\mathcal{T}\left(\ell_{x, p, L}, D^{o}\right) \subset S_{x, p, L}$ and while $\mathcal{T}\left(\ell_{x, p, L}, D^{o}\right) \not \subset D^{o}$ it is the case that $\mathcal{T}\left(\ell_{x, p, L}, D^{o}\right) \subset$ $D^{o,+1}$.

The key lemma used for the comparison of the stick process and the oriented percolation model considers the set of line segments $\ell_{y, q, L}$ such that $S_{y, q, L} \cap \mathcal{T}\left(\ell_{x, p, L}, D^{o}\right) \neq \emptyset$ and such that $\ell_{y, q, L} \cap B\left(\zeta_{y}, 2\right) \neq \emptyset$ for some $\zeta_{y} \in T^{-16}\left(D^{(0,2)}\right)$. That is, the corresponding stick $S_{y, q, L}$ connects with the target, and also presents a new (vertical) target in the box $D^{(0,2)}$ that can be used for further connections (see Fig. 1). The proof of this lemma considers two collections of balls, and the proof will use the elementary case in Lemma 5.1 (which concerns only two balls). The first collection will be embedded in the target, while the second will be a collection with centers embedded in $T^{-16}\left(D^{(0,2)}\right)$. Then, we will sum over these collections to obtain our estimate. For further steps in the construction, we will need to define collections with centers in $L^{-16}\left(D^{(0,2)}\right), R^{-16}\left(D^{(0,2)}\right)$ and $T^{-16}\left(D^{(0,2)}\right)$ respectively as follows. Let

$$
\begin{aligned}
& \mathbb{L}\left(D^{u}\right)=\left\{x \in L^{-16}\left(D^{u}\right):\left(x_{2}, \ldots, x_{d}\right) \in 12 \mathbb{Z}^{d-1}\right\}, \\
& \mathbb{R}\left(D^{u}\right)=\left\{x \in R^{-16}\left(D^{u}\right):\left(x_{2}, \ldots, x_{d}\right) \in 12 \mathbb{Z}^{d-1}\right\} \text { and } \\
& \mathbb{T}\left(D^{u}\right)=\left\{x \in T^{-16}\left(D^{u}\right):\left(x_{1}, x_{3}, \ldots, x_{d}\right) \in 12 \mathbb{Z}^{d-1}\right\} .
\end{aligned}
$$

We see that these are discretizations of their respective boundary pieces with points separated by a distance of at least 12 . The reason for this separation distance is that certain hitting events will become independent. In order to show this, we will need a "disjointness" result, namely Lemma 5.2 whose proof is provided in Appendix A.3. We also note for future reference that the number of points $\left|\mathbb{T}\left(D^{u}\right)\right|$ in $\mathbb{T}\left(D^{u}\right)$ can be bounded below by

$$
\left|\mathbb{T}\left(D^{u}\right)\right| \geq\left(\frac{\frac{L}{8 \sqrt{d}}-32}{12}-1\right)^{d-1} \geq\left(\frac{L}{96 \sqrt{d}}-4\right)^{d-1},
$$

since the side length of $T^{-16}\left(D^{u}\right)$ is $\frac{L}{8 \sqrt{d}}-32$, the spacing of the lattice is 12 , and then we subtract 1 for boundary issues.

Similar to the definition of $\ell_{x, p, L}$, let

$$
\ell_{x, p, \infty}=\{x+t p:-\infty<t<\infty\}
$$

and

$$
\ell_{y, q, \infty}=\{y+\tau q:-\infty<\tau<\infty\},
$$

where $p, q$ as before are vectors such that $\|p\|=\|q\|=1$, be two parametrized (infinite) lines. Informally, Lemma 5.2 shows that if the angle between two orientation vectors $p$ and $q$ is not too small (or equivalently if $|\langle p, q\rangle|$ is not too large), then the lines $\ell_{x, p, \infty}$ and $\ell_{x, q, \infty}$ will not be close for very long.

Lemma 5.2 Assume that $p, q \in \mathbb{S}$ are such that $|\langle p, q\rangle| \leq \frac{1}{\sqrt{2}}$ and that $t_{1}, \tau_{1}$ are such that

$$
\left\|\ell_{x, p, \infty}\left(t_{1}\right)-\ell_{y, q, \infty}\left(\tau_{1}\right)\right\| \leq 2 .
$$

Then we have that for every $(t, \tau)$ such that $\max \left(\left|t-t_{1}\right|,\left|\tau-\tau_{1}\right|\right) \geq 12$,

$$
\left\|\ell_{x, p, \infty}(t)-\ell_{y, q, \infty}(\tau)\right\| \geq 6 .
$$


Remark If $|\langle p, q\rangle| \leq 1 / \sqrt{2}$, and if $B\left(\ell_{y, q, \infty}\left(\tau_{1}\right), 2\right)$ is such that $\ell_{x, p, \infty} \cap B\left(\ell_{y, q, \infty}\left(\tau_{1}\right), 2\right) \neq$ $\emptyset$, then for some $t_{1}$,

$$
\left\|\ell_{x, p, \infty}\left(t_{1}\right)-\ell_{y, q, \infty}\left(\tau_{1}\right)\right\| \leq 2 .
$$

It follows from Lemma 5.2 that if $\left|\tau_{1}-\tau_{2}\right| \geq 12$, then for every value of $t$,

$$
\left\|\ell_{x, p, \infty}(t)-\ell_{y, q, \infty}\left(\tau_{2}\right)\right\| \geq 6 .
$$

We therefore see that

$$
\ell_{x, p, \infty} \cap B\left(\ell_{y, q, \infty}\left(\tau_{2}\right), 2\right)=\emptyset .
$$

Informally, this means that if the line $\ell_{x, p, \infty}$ hits a ball of radius 2 centered on the line $\ell_{y, q, \infty}$, it can not hit any other such ball as long as the distance between the centers exceed 12 . This is how Lemma 5.2 will be used.

We can now state and prove our key lemma.

Lemma 5.3 Let $\gamma_{x} \in D^{(-2,0)}, \zeta_{x} \in \mathbb{R}\left(D^{o}\right)$ and $\ell_{x, p, L}$ be such that

$$
B\left(\gamma_{x}, 2\right) \stackrel{\ell_{x, p, L}}{\longleftrightarrow} B\left(\zeta_{x}, 2\right) .
$$

For every $d \geq 2, \gamma_{x}, \zeta_{x}$ and $\ell_{x, p, L}$ as above, we have that

$$
\begin{aligned}
& \mathbb{P}\left(\exists(y, q) \in \Pi^{\lambda}, \zeta_{y} \in \mathbb{T}\left(D^{(0,2)}\right): y \in D^{(0,1)}, \mathcal{T}\left(\ell_{x, p, L}, D^{o}\right)^{+1} \stackrel{\ell_{y, q, L}}{\longleftrightarrow} B\left(\zeta_{y}, 2\right)\right) \\
& \quad \geq 1-\frac{1}{c_{d}^{\prime} \lambda \delta L^{2}},
\end{aligned}
$$

for $L>200 \sqrt{d}$ and where

$$
c_{d}^{\prime}=\frac{c_{d}}{(1000 \sqrt{d})^{d}}
$$

with $c_{d}$ is as in Lemma 5.1.

Proof As mentioned, the proof will rely on Lemma 5.1 and two collections of balls. Our first collection is simply all $B(z, 2)$ where $z \in \mathbb{T}\left(D^{(0,2)}\right)$. In order to find our second collection, we start by fixing some $\gamma_{x}, \zeta_{x}$ and $\ell_{x, p, L}$ as in the assumption. Then, let $t_{1}<t_{2}<\cdots<t_{N}$ be such that $\ell_{x, p, L}\left(t_{i}\right) \in D^{o}$ for every $i=1, \ldots, N$, and such that $\left|t_{i}-t_{i+1}\right|=12$ for every $i=1, \ldots, N-1$. Next, consider any $z=\left(z_{1}, \ldots, z_{d}\right) \in \ell_{x, p, L}$ such that

$$
z_{1} \in\left[-\frac{L}{16 \sqrt{d}}, \frac{L}{16 \sqrt{d}}\right] .
$$

It is a straightforward, although somewhat tedious, exercise in trigonometry to show that since $\ell_{x, p, L} \cap D^{(-2,0),+2} \neq \emptyset$ and $\ell_{x, p, L} \cap B\left(\zeta_{x}, 2\right) \neq \emptyset$, we must for such a $z$ have that

$$
\left|z_{k}\right| \leq \frac{L}{16 \sqrt{d}} \text { for } k=1,2, \ldots, d .
$$

We will leave the details of this fact to the reader, although we remark that we outline the argument for a similar statement in the proof of Lemma 5.1 in Appendix A.2. Thus, if $z \in \ell_{x, p, L}$ satisfies (5.8) it follows from (5.9) that $z \in D^{o}$, and so we conclude that the length 
of $\ell_{x, p, L} \cap D^{o}$ must be at least $\frac{L}{8 \sqrt{d}}$. (Observe that if we would not have required $\ell_{x, p, L}$ to hit "well inside" the right boundary of $D^{o}$, i.e. $R^{-16}\left(D^{o}\right)$, then $\ell_{x, p, L}$ could potentially have missed most or all of $D^{o}$.) From this it then follows similarly to (5.6) that we may take

$$
N \geq \frac{1}{12} \frac{L}{8 \sqrt{d}}-1=\frac{L}{96 \sqrt{d}}-1 \geq \frac{L}{1000 \sqrt{d}},
$$

which holds since we assume that $L>200 \sqrt{d}$. Informally, this simply means that one can find a string of order $L$ balls within the target $\mathcal{T}\left(\ell_{x, p, L}, D^{o}\right)$ such that the distance between consecutive centers of these balls is always 12. This is our second collection of balls.

Our next step is to prove that

$$
\left\{\ell_{y, q, L}: B\left(t_{i}, 2\right) \stackrel{\ell_{y, q, L}}{\longleftrightarrow} B(z, 2)\right\} \cap\left\{\ell_{y, q, L}: B\left(t_{j}, 2\right) \stackrel{\ell_{y, q, L}}{\longleftrightarrow} B(w, 2)\right\}=\emptyset
$$

whenever either $i \neq j$, or $z, w \in \mathbb{T}\left(D^{(0,2)}\right)$ is such that $z \neq w$, or both. We will show this for $i \neq j$ and for $z=w$. All other cases follow in the same way. Therefore, assume that $\ell_{y, q, L} \cap B(z, 2) \neq \emptyset$ and that $\ell_{y, q, L} \cap B\left(t_{i}, 2\right) \neq \emptyset$. We claim that $\ell_{y, q, L} \cap B\left(t_{j}, 2\right)=\emptyset$ for $j \neq i$. The key to showing this claim lies in analyzing the orientation vectors $p, q$ and then using Lemma 5.2 (see also the remark after the statement of that lemma). In order to use Lemma 5.2 we need to prove that $|\langle p, q\rangle| \leq \frac{1}{\sqrt{2}}$. We will do this by showing that $\left|p_{k}\right|$ is small for $k=2, \ldots, d$ and that $\left|q_{k}\right|$ is small for $k=1,3, \ldots, d$.

Recall that $\ell_{x, p, L} \cap B\left(\gamma_{x}, 2\right) \neq \varnothing$ and $\ell_{x, p, L} \cap B\left(\zeta_{x}, 2\right) \neq \emptyset$ where $\gamma_{x} \in D^{(-2,0)}$ and $\zeta_{x} \in \mathbb{R}\left(D^{o}\right)$. Therefore, we can write

$$
p=\frac{\gamma-\zeta}{\|\gamma-\zeta\|}
$$

for some $\gamma \in B\left(\gamma_{x}, 2\right)$ and $\zeta \in B\left(\zeta_{x}, 2\right)$. If we let $\xi=\left(\xi_{1}, \ldots, \xi_{d}\right)=\gamma-\zeta$ we then see that

$$
2 \frac{L}{4}-4 \leq\left|\xi_{1}\right| \leq 2 \frac{L}{4}+\frac{L}{8 \sqrt{d}}+4 \text { and that }\left|\xi_{k}\right| \leq \frac{L}{8 \sqrt{d}}-12
$$

for $k=2, \ldots, d$. It follows that $\|\xi\| \geq \frac{L}{2}-4$ and so we conclude that

$$
\left|p_{k}\right|=\frac{\left|\xi_{k}\right|}{\|\xi\|} \leq \frac{\frac{L}{8 \sqrt{d}}-12}{\frac{L}{2}-4} \leq \frac{1}{4 \sqrt{d}}
$$

for $k=2, \ldots, d$ and every $d \geq 2\left(\right.$ where of course $p=\left(p_{1}, \ldots, p_{d}\right)$ ).

Again by assumption, $\ell_{y, q, L} \cap B\left(t_{i}, 2\right) \neq \emptyset$ and $\ell_{y, q, L} \cap B(z, 2) \neq \emptyset$ where $z \in \mathbb{T}\left(D^{(0,2)}\right)$. Therefore, we can write

$$
q=\frac{\tilde{\gamma}-\tilde{\zeta}}{\|\tilde{\gamma}-\tilde{\zeta}\|}
$$

for some $\tilde{\gamma} \in B\left(t_{i}, 2\right)$ and $\tilde{\zeta} \in B(z, 2)$. As for $p$, it follows here that

$$
\left|q_{k}\right| \leq \frac{1}{4 \sqrt{d}}
$$


for $k=1,3, \ldots, d$ (since $q$ points almost vertically and $p$ points almost horizontally). Therefore we can conclude that

$$
\begin{aligned}
& |\langle p, q\rangle| \leq\left|p_{1}\right| \cdot\left|q_{1}\right|+\cdots+\left|p_{d}\right| \cdot\left|q_{d}\right| \\
& \quad \leq\left|q_{1}\right|+\left|p_{2}\right|+\left|p_{3}\right| \cdot\left|q_{3}\right|+\cdots+\left|p_{d}\right| \cdot\left|q_{d}\right| \\
& \quad \leq \frac{1}{4 \sqrt{d}}+\frac{1}{4 \sqrt{d}}+(d-2) \frac{1}{16 d} \leq \frac{1}{\sqrt{2}},
\end{aligned}
$$

for every $d \geq 2$. It follows from Lemma 5.2 and the remark thereafter that $\ell_{y, q, L} \cap B\left(t_{j}, 2\right)=$ $\emptyset$ since $\left|t_{i}-t_{j}\right| \geq 12$.

We can now conclude from Lemma 5.1 that

$$
\begin{aligned}
& \mu_{\lambda}\left((y, q): y \in D^{(0,1)}, \mathcal{T}\left(\ell_{x, p, L}, D^{o}\right)^{+1} \stackrel{\ell_{y, q, L}}{\longleftrightarrow} B(z, 2) \text { for some } z \in \mathbb{T}\left(D^{(0,2)}\right)\right) \\
& \geq \mu_{\lambda}\left((y, q): y \in D^{(0,1)}, B\left(t_{i}, 2\right) \stackrel{\ell_{y, q, L}}{\longleftrightarrow} B(z, 2),\right. \\
& \text { for some } \left.i=1, \ldots, N \text { and } z \in \mathbb{T}\left(D^{(0,2)}\right)\right) \\
& =\sum_{i=1}^{N} \sum_{z \in \mathbb{T}\left(D^{(0,2)}\right)} \mu_{\lambda}\left((y, q): y \in D^{(0,1)}, B\left(t_{i}, 2\right) \stackrel{\ell_{y, q, L}}{\longleftrightarrow} B(z, 2)\right) \\
& \geq N\left|\mathbb{T}\left(D^{(0,2)}\right)\right| \lambda \delta c_{d} L^{-d+2}
\end{aligned}
$$

where the disjointness was used in the first equality and Lemma 5.1 in the second inequality. It follows from (5.6) that

$$
\left|\mathbb{T}\left(D^{(0,2)}\right)\right| \geq\left(\frac{L}{96 \sqrt{d}}-4\right)^{d-1} \geq\left(\frac{L}{1000 \sqrt{d}}\right)^{d-1},
$$

since $L>200 \sqrt{d}$. Furthermore, by also using (5.10) we conclude that

$$
\begin{aligned}
& N\left|\mathbb{T}\left(D^{(0,2)}\right)\right| \lambda \delta c_{d} L^{-d+2} \\
& \geq \frac{L}{1000 \sqrt{d}}\left(\frac{L}{1000 \sqrt{d}}\right)^{d-1} \lambda \delta c_{d} L^{-d+2}=\lambda \delta \frac{c_{d}}{(1000 \sqrt{d})^{d}} L^{2}=\lambda \delta c_{d}^{\prime} L^{2} .
\end{aligned}
$$

Combining (5.11) and (5.12), we then see that

$$
\begin{aligned}
& \mu_{\lambda}\left((y, q): y \in D^{(0,1)}, \mathcal{T}\left(\ell_{x, p, L}, D^{o}\right)^{+1} \stackrel{\ell_{y, q, L}}{\longleftrightarrow} B(z, 2) \text { for some } z \in \mathbb{T}\left(D^{(0,2)}\right)\right) \\
& \geq \lambda \delta c_{d}^{\prime} L^{2} .
\end{aligned}
$$

Therefore,

$$
\begin{aligned}
& \mathbb{P}\left(\exists(y, q) \in \Pi^{\lambda}, z \in \mathbb{T}\left(D^{(0,2)}\right): y \in D^{(0,1)}, \mathcal{T}\left(\ell_{x, p, L}, D^{o}\right)^{+1} \stackrel{\ell_{y, q, L}}{\longleftrightarrow} B(z, 2)\right) \\
& \quad=1-\exp \left(-\mu_{\lambda}\left((y, q): y \in D^{(0,1)},\right.\right. \\
& \left.\left.\mathcal{T}\left(\ell_{x, p, L}, D^{o}\right)^{+1} \stackrel{\ell_{y, q, L}}{\longleftrightarrow} B(z, 2) \text { for some } z \in \mathbb{T}\left(D^{(0,2)}\right)\right)\right) \\
& \quad \geq 1-e^{-\lambda \delta c_{d}^{\prime} L^{2}} \geq 1-\frac{1}{c_{d}^{\prime} \lambda \delta L^{2}},
\end{aligned}
$$


where we used that $e^{-x} \leq x^{-1}$ for every $x>0$.

We are now ready to prove the upper bound of Theorem 3.1.

Proof of upper bound of Theorem 3.1 Consider the bond version of the oriented percolation model described at the beginning of this section and recall the notation $\left(A_{n}\right)_{n \geq 0}$. Recall also that we will here work on the lattice $2 \mathbb{H}$ so that $A_{n} \subset\left\{u \in \mathbb{H}: u_{1}=2 n\right\}$. In our construction below, we will consider a sequence of random sets $\left(E_{n}\right)_{n \geq 0}$ defined by letting $u \in E_{n}$ if there exists a "good" path of sticks connecting a base stick $S_{x, p, L}$ to the box $D^{u}$. Our coupling will yield $A_{n} \subset E_{n}$ for every $n \geq 1$, where the value of $\alpha$ for the oriented percolation model will depend on $\lambda$. We will then show that for $\lambda$ larger than the upper bound of the statement of this theorem, we will have that $\alpha \geq 0.81$ and therefore (5.3) is satisfied. This then shows that there exists an unbounded connected component in $\mathcal{C}\left(\Pi^{\lambda}\right)$ with positive probability. We choose to work with $\alpha \geq 0.81$ rather than $\alpha>2 / 3$ out of convenience, as making a more optimal choice of $\alpha$ would not affect the quality of our bound in any meaningful way.

We will now fix

$$
\lambda>\frac{10}{9 \delta c_{d}^{\prime}} L^{-2}=\frac{10(1000 \sqrt{d})^{d}}{9 \delta c_{d}} L^{-2}=\frac{10(1000 \sqrt{d})^{d} \sqrt{d} \Gamma(2 d-1)}{9 \delta 2^{5(d-2)} \pi^{d / 2-2} \Gamma(d / 2)^{3}} L^{-2},
$$

so that $\lambda$ is above $1 / 2$ of the upper bound in the statement. It will be convenient to use two independent Poisson point processes $\Pi_{1}^{\lambda}$ and $\Pi_{2}^{\lambda}$, defined on the same probability space and with the same distribution as $\Pi^{\lambda}$. Clearly, if $\Pi_{1}^{\lambda}$ and $\Pi_{2}^{\lambda}$ are independent, $\Pi_{1}^{\lambda}+\Pi_{2}^{\lambda}$ is equal to $\Pi^{2 \lambda}$ in distribution (this is why the right-hand side of (5.13) equals $1 / 2$ of the upper bound of the statement).

We will proceed with our construction below in steps. The general idea is illustrated in Fig. 3, and it may be useful to consult this when reading what follows.

In Step 0, observe that the event that there exists some

$$
(x, p) \in \Pi_{1}^{\lambda} \text { such that } D^{(-2,0),+2} \stackrel{\ell_{x, p, L}}{\longleftrightarrow} R^{-16}\left(D^{o}\right),
$$

has positive probability for every $\lambda>0$. We therefore condition on this event, and let $E_{0}=\{o\}$. Observe that $A_{0}=E_{0}$.

Next, for Step 1-a we observe that by Lemma 5.3 we have that

$$
\begin{array}{r}
\mathbb{P}\left(\exists\left(y_{v}, q_{v}\right) \in \Pi_{1}^{\lambda}, \zeta_{y_{v}} \in \mathbb{T}\left(D^{(0,2)}\right): y_{v} \in D^{(0,1)}, \mathcal{T}\left(\ell_{x, p, L}, D^{o}\right)^{+1} \stackrel{\left.\stackrel{\ell_{y_{v}, q_{v}, L}}{\longleftrightarrow} B\left(\zeta_{y_{v}}, 2\right)\right)}{\geq 1-\frac{1}{\lambda \delta c_{d}^{\prime} L^{2}},}\right.
\end{array}
$$

where $c_{d}^{\prime}$ is as in that lemma. Using obvious notation we then let $\mathcal{T}\left(\ell_{y_{v}, q_{v}, L}, D^{(0,2)}\right)$ denote the new, almost-vertical target provided by $\ell_{y_{v}, q_{v}, L}$. Conditioned on the existence of such a line $\ell_{y_{v}, q_{v}, L}$ we can again use Lemma 5.3 along with rotational invariance to see that

$$
\begin{aligned}
& \mathbb{P}\left(\exists\left(y_{h}, q_{h}\right) \in \Pi_{1}^{\lambda}, \zeta_{y_{h}} \in \mathbb{R}\left(D^{(2,2)}\right): y_{h} \in D^{(1,2)}, \mathcal{T}\left(\ell_{y_{v}, q_{v}, L}, D^{(0,2)}\right)^{+1} \stackrel{\ell_{y_{h}, q_{h}, L}}{\longrightarrow} B\left(\zeta_{y_{h}}, 2\right)\right) \\
& \quad \geq 1-\frac{1}{\lambda \delta c_{d}^{\prime} L^{2}},
\end{aligned}
$$

which is Step 1-b. Since Step 1-a concerns line segments with centers $y_{v} \in D^{(0,1)}$ and Step 1 -b concerns line segments with centers $y_{h} \in D^{(1,2)}$, we conclude that the probability that 


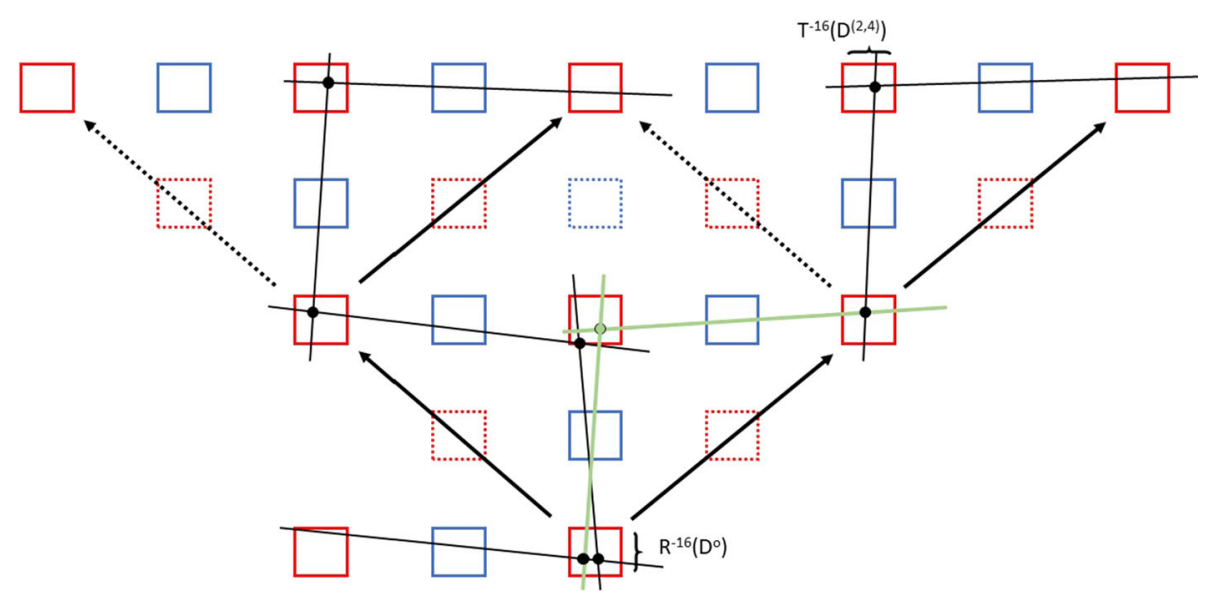

Fig. 3 An illustration of the coupling with oriented percolation. The red boxes are target boxes, while the blue boxes are center boxes (i.e. where the centers of the line segments belong). At the bottom is a line segment with center in $D^{(-1,0)}$ and which hits the target face $R^{-16}\left(D^{o}\right)$. The two sticks that are green (the color is only there for explanatory purposes) are the ones found during Step 1-a and 1-b, and together they constitute a good right-oriented path from $\mathcal{T}\left(\ell_{x, p, L}, D^{o}\right)$ to $D^{(2,2)}$. We can also see a good left-oriented path from $\mathcal{T}\left(\ell_{x, p, L}, D^{o}\right)$ to $D^{(-2,2)}$. These good paths induce connections from $o$ to $(-2,2)$ and $(2,2)$ in the oriented percolation model which are indicated by solid arrows. We also see connections from $(2,2)$ to $(4,4)$ and from $(-2,2)$ to $(0,4)$. However there are no connection from $(2,2)$ to $(0,4)$ nor from $(-2,2)$ to $(-4,4)$ (indicated by dashed, i.e. missing arrows). Observe the line centered in $D^{(-1,2)}$, hitting the target in $D^{(0,2)}$ (and then proceeding to $\left.D^{(-2,2)}\right)$ but which does not hit $R^{-16}\left(D^{(0,2)}\right)$. Note however that (as required) it does hit $L^{-16}\left(D^{(-2,2)}\right)$. Lastly, the dashed boxes are not used in the construction

there exists a "right-oriented" (see the green lines in Fig. 3) path from $\mathcal{T}\left(\ell_{x, p, L}\right)$ to some $B\left(\zeta_{y_{h}}, 2\right)$ where $\zeta_{y_{h}} \in \mathbb{R}\left(D^{(2,2)}\right)$, is at least

$$
\left(1-\frac{1}{\lambda \delta c_{d}^{\prime} L^{2}}\right)^{2} \geq\left(1-\frac{1}{\frac{10}{9 \delta c_{d}^{\prime}} L^{-2} c_{d}^{\prime} \delta L^{2}}\right)^{2}=\left(1-\frac{9}{10}\right)^{2}=0.81,
$$

by using (5.13). Note that the corresponding sticks $S_{x, p, L}, S_{y_{v}, q_{v}, L}$ and $S_{y_{h}, q_{h}, L}$ form a connected path and we call such a path a good path. Note also that the line $y_{h, q, L}$ presents a new target $\mathcal{T}\left(\ell_{y_{h}, q, L}, D^{(2,2)}\right)$ from which the construction can proceed. If a good rightoriented path as described exists, we let $(2,2) \in E_{1}$ and because of (5.14) we can couple this with $A_{1}$ so that $(2,2) \in A_{1}$ with probability 0.81 .

In the same way, we explore $\Pi_{2}^{\lambda}$ and attempt to find a left oriented good path from $D^{o}$ to $D^{(-2,2)}$. Indeed, as above, the probability that there exists a good path within $\Pi_{2}^{\lambda}$ from $\mathcal{T}\left(\ell_{x, p, L}, D^{o}\right)$ via $D^{(0,2)}$ to some $B\left(\zeta_{h}^{\prime}, 2\right)$ where $\zeta_{h}^{\prime} \in \mathbb{L}\left(D^{(-2,2)}\right)$, and where the last line $y_{h, q, L}^{\prime}$ presents a new target $\mathcal{T}\left(\ell_{y_{h}^{\prime}, q, L}, D^{(-2,2)}\right)$ is by symmetry at least $\alpha$. We say that $(-2,2) \in E_{1}$ if such a left oriented path exists. Furthermore, since $\Pi_{1}^{\lambda}$ and $\Pi_{2}^{\lambda}$ are independent, the existence of the right-oriented and the left-oriented paths are also independent, and so the events $\left\{u \in E_{1}\right\}$ for $u \in\{(-2,2),(2,2)\}$ are conditionally independent conditioned on the existence of $\mathcal{T}\left(\ell_{x, p, L}, D^{o}\right)$. Using (5.14), we can couple this described procedure with the oriented percolation model with parameter $\alpha=0.81$ in such a way that if there is an arrow from $o$ to $(-2,2)$ in the oriented percolation model, then there is a good path from $\mathcal{T}\left(\ell_{x, p, L}\right)$ to $D^{(-2,2)}$ in the stick process. In the same way, if there is an arrow from $o$ to 
$(2,2)$ in the oriented percolation model, there is also a good path from $\mathcal{T}\left(\ell_{x, p, L}\right)$ to $D^{(2,2)}$ in the stick model. For our next step, we let $\mathcal{T}_{1}=\left\{\mathcal{T}^{u}\right\}_{u \in E_{1}}$ be the set of targets presented by the paths we found in Step 1. Our coupling shows that $A_{1} \subset E_{1}$, and we note that there is a path of sticks from $D^{o}$ to $D^{u}$ for every $u \in E_{1}$ within $\mathcal{C}\left(\Pi^{2 \lambda}\right)$ where $\Pi^{2 \lambda}=\Pi_{1}^{\lambda}+\Pi_{2}^{\lambda}$.

Step 2 works in much the same way as Step 1, and so we condition on $E_{1}$ and $\mathcal{T}_{1}$ from Step 1. Given a target $\mathcal{T}^{u} \in \mathcal{T}_{1}$, we use $\Pi_{1}^{\lambda}$ to attempt to find a good right-oriented path from the target $\mathcal{T}^{u} \in \mathcal{T}_{1}$ to $D^{u+(2,2)}$ using two sticks with centers in $D^{u+(0,1)}$ and $D^{u+(1,2)}$ respectively. Furthermore, we use $\Pi_{2}^{\lambda}$ when attempting to find a left-oriented path from $\mathcal{T}^{u} \in \mathcal{T}_{1}$ to $D^{u+(-2,2)}$ using two sticks with centers in $D^{u+(0,1)}$ and $D^{u+(-1,2)}$ respectively. Note that the target $\mathcal{T}^{u}$ can be a result of Step 1 yielding a good path using $\Pi_{1}^{\lambda}$, but that we attempt to find a good path to $D^{u+(-2,2)}$ using $\Pi_{2}^{\lambda}$. This is not an issue as we in the end consider $\Pi_{1}^{\lambda}+\Pi_{2}^{\lambda}$ (or rather the union of all sticks associated to points from $\Pi_{1}^{\lambda}$ or $\Pi_{2}^{\lambda}$, i.e. $\left.\mathcal{C}\left(\Pi^{2 \lambda}\right)\right)$. We note further that if $E_{1}=\{(-2,2),(2,2)\}$, then there can be a good path both from $(-2,2)$ to $(0,4)$ and from $(2,2)$ to $(0,4)$. If both of these events occur, then there are two possible targets in $D^{(0,4)}$ for Step 3. In order to avoid ambiguities, we will in this and all similar cases always use the target $\mathcal{T}^{(0,4)}$ provided by the configuration $\Pi_{1}^{\lambda}$ from the previous step (in this case Step 2). Since finding a good path always has probability at least $\alpha$, we can couple $A_{2}, E_{2}$ such that $A_{2} \subset E_{2}$. As before, we let $\mathcal{T}_{2}=\left\{\mathcal{T}^{u}\right\}_{u \in E_{2}}$ be the set of targets provided by the good paths.

The general step is now clear. Given $E_{n}$ and the targets $\mathcal{T}_{n}$ from Step $n$, we look for good paths from $D^{u}$ to $D^{u+(2,2)}$ using $\Pi_{1}^{\lambda}$, and from $D^{u}$ to $D^{u+(-2,2)}$ using $\Pi_{2}^{\lambda}$. With this coupling we see that $A_{n} \subset E_{n}$ for every $n \geq 0$, and since we chose $\alpha=0.81$ so that (5.3) holds, we conclude that

$$
\mathbb{P}\left(E_{n} \neq \emptyset \quad \forall n \geq 0\right)>0,
$$

for $\lambda$ as in (5.13). Clearly, if the event $\left\{E_{n} \neq \emptyset \forall n \geq 0\right\}$ occurs, then $S_{x, p, L}$ belongs to an unbounded connected component, and so $\mathcal{C}\left(\Pi^{2 \lambda}\right)$ percolates.

\subsection{The Upper Bound of Theorem 3.2}

We now turn to the proof of the upper bound of Theorem 3.2. Again, we will couple our stick process with oriented percolation, but this time to the site percolation model on $\mathbb{H}$.

Proof of upper bound of Theorem 3.2 In the rigid case we have that $p=e_{2}$ with probability one, and so we will simply write $\left(x, e_{2}\right)$.

Similar to the notation $D^{u}$, for $u \in \mathbb{H}$, we let

$$
B^{u}(1 / 2):=B\left(\left(u_{1},(L / 2+2) u_{2}, 0, \ldots, 0\right), 1 / 2\right),
$$

so that $B^{u}(1 / 2) \subset \mathbb{R}^{d}$ is a closed ball of radius $1 / 2$ corresponding to the point $u \in \mathbb{H}$.

For $u \in \mathbb{H}$ such that $u_{2} \geq 1$, we now consider the event

$$
\begin{aligned}
& G_{u}=\left\{\omega \in \Omega: \exists\left(x, e_{2}\right) \in \omega \text { such that } B^{u}(1 / 2) \subset S_{x, e_{2}, L},\right. \\
& \left.B^{u+(-1,-1)}(1 / 2) \cap S_{x, e_{2}, L} \neq \emptyset \text { and } B^{u+(1,-1)}(1 / 2) \cap S_{x, e_{2}, L} \neq \emptyset\right\} .
\end{aligned}
$$

The event $G_{u}$ implies that the ball $B^{u}(1 / 2)$ is completely covered by a stick, while the same stick intersects the two balls to the lower left and right (see Fig. 4a). Furthermore, we note that in order for a stick $S_{x, e_{2}, L}$ to contain both $B^{u}(1 / 2)$ and $B^{u+(-1,-1)}(1 / 2)$, it must be that $x_{2}=u_{2}-1 / 2$. This event has measure 0 . Furthermore, since the centers of $B^{u}(1 / 2)$ and $B^{u+(0,2)}(1 / 2)$ are at distance $L+4$, no stick can contain both of these balls. It follows that 


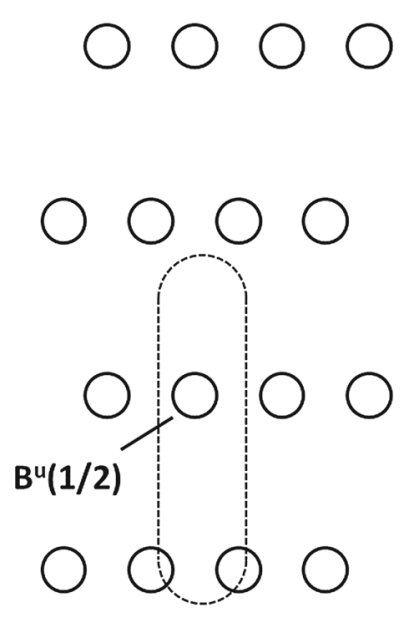

(a)

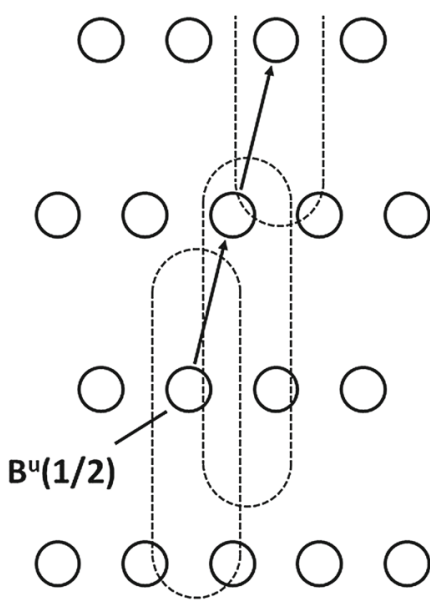

(b)

Fig. 4 In a the event $G_{u}$ is illustrated. The ball corresponding to $u \in \mathbb{H}$, i.e. $B^{u}(1 / 2)$, is completely covered by the stick (dashed). Furthermore, the stick intersects the two balls $B^{u+(-1,-1)}(1 / 2)$ and $B^{u+(1,-1)}(1 / 2)$ one row below. In $\mathbf{b}$ the events $G_{u}, G_{u+(1,1)}$ and $G_{u+(2,2)}$ all occur. We see that the corresponding sticks are part of the same connected component

outside of an event of measure 0 , a stick can only contain one ball, and therefore the events $\left\{G_{u}\right\}_{u \in \mathbb{H}, u_{2} \geq 1}$ are independent. Furthermore, we note that if $\Pi^{\lambda} \in G_{u} \cap G_{u+(-1,-1)}$, then the corresponding sticks which contain $B^{u}(1 / 2)$ and $B^{u+(-1,-1)}(1 / 2)$ respectively must touch, and that they therefore belong to the same connected component of $\mathcal{C}\left(\Pi^{\lambda}\right)$ (see also Fig. 4b). We can use these properties to couple the stick process with the site percolation variant of the oriented percolation model as we now explain.

First, it is clear that

$$
\mathbb{P}\left(\exists\left(x, e_{2}\right) \in \Pi^{\lambda}: B^{o}(1 / 2) \subset S_{x, e_{2}, L}\right)>0,
$$

and if the event in this probability occurs, we set $E_{0}=A_{0}=\{o\}$. Then, conditioned on $E_{0}=\{o\}$, we let

$$
E_{1}=\left\{u \in \mathbb{H}: \Pi^{\lambda} \in G_{u}\right\} \cap\{u \in\{(-1,1),(1,1)\}\},
$$

and we see that $\mathbb{P}\left(u \in E_{1} \mid E_{0}\right)=\mathbb{P}\left(G_{u}\right)$ and that the events $(-1,1) \in E_{1}$ and $(1,1) \in E_{1}$ are conditionally independent. In general we let

$$
E_{n}=\left\{u \in \mathbb{H}: \Pi^{\lambda} \in G_{u}\right\} \cap\left\{u \in\left\{u^{\prime}+(1,-1), u^{\prime}+(1,1)\right\}, \text { where } u^{\prime} \in E_{n-1}\right\},
$$

and again we see that $\mathbb{P}\left(u \in E_{n} \mid E_{n-1}\right)=\mathbb{P}\left(G_{u}\right)$ for any $u$ such that $u \in\left\{u^{\prime}+(-1,1), u^{\prime}+\right.$ $(1,1)\}$, where $u^{\prime} \in E_{n-1}$. Conditional independence is also clear. We see that this is indeed the site percolation version of the oriented percolation model, since both probabilities in the two first rows of (5.2) are $\mathbb{P}\left(G_{u}\right)$ in our case. Since this version survives with positive probability if $\alpha>3 / 4$ (recall the discussion at the start of Sect. 5) we need to show that $\mathbb{P}\left(G_{u}\right)>3 / 4$ whenever

$$
\lambda>4 \frac{2^{d} \Gamma((d+1) / 2)}{\pi^{d / 2-1}} L^{-1} .
$$


In order to bound $\mathbb{P}\left(G_{u}\right)$ we consider, without loss of generality, the case where $u=(1,1)$. Recall that the center of $B^{(1,1)}(1 / 2)$ is at the point $(1,(L / 2+2), 0, \ldots, 0)$, and observe that in order for $B^{(1,1)}(1 / 2) \subset S_{x, e_{2}, L}$ it is sufficient that $\left\|\left(x_{1}, x_{3}, \ldots, x_{d}\right)-(1,0, \ldots, 0)\right\|=$ $\left\|\left(x_{1}-1, x_{3}, \ldots, x_{d}\right)\right\| \leq 1 / 2$ and that $x_{2} \in[2, L+2]$. Next, in order for $G_{u}$ to occur, we must also have that $G^{o}(1 / 2) \cap S_{x, e_{2}, L} \neq \emptyset$. For this, it is sufficient that $\left\|\left(x_{1}, x_{3}, \ldots, x_{d}\right)\right\| \leq$ $3 / 2$ and that $x_{2} \in[-L / 2, L / 2]$. Similarly, in order for $G^{(2,0)}(1 / 2) \cap S_{x, e_{2}, L} \neq \varnothing$ it is sufficient that $\left\|\left(x_{1}, x_{3}, \ldots, x_{d}\right)-(2,0, \ldots, 0)\right\|=\left\|\left(x_{1}-2, x_{3}, \ldots, x_{d}\right)\right\| \leq 3 / 2$ and that $x_{2} \in[-L / 2, L / 2]$. We then observe that all of these three conditions are satisfied whenever

$$
\left\|\left(x_{1}-1, x_{3}, \ldots, x_{d}\right)\right\| \leq 1 / 2 \text { and } x_{2} \in[2, L / 2] .
$$

Therefore (recall the notation $B_{d-1}(x, r)$ from Sect. 2),

$$
\begin{aligned}
& \mu_{\lambda}\left(\left(x, e_{2}\right): B^{(1,1)}(1 / 2) \subset S_{x, e_{2}, L}, B^{o}(1 / 2) \cap S_{x, e_{2}, L} \neq \emptyset\right. \\
& \left.\quad \text { and } B^{(2,0)}(1 / 2) \cap S_{x, e_{2}, L} \neq \emptyset\right) \\
& =\lambda \int_{x \in \mathbb{R}^{d}} I\left(\left\|\left(x_{1}-1, x_{3}, \ldots, x_{d}\right)\right\| \leq 1 / 2 \text { and } x_{2} \in[2, L / 2]\right) \mathrm{d} x \\
& =\lambda(L / 2-2) \operatorname{Vol}\left(B_{d-1}(o, 1 / 2)\right)=\lambda(L / 2-2) \frac{\pi^{(d-1) / 2}}{2^{d-1} \Gamma((d+1) / 2)} \\
& >\lambda L \frac{\pi^{d / 2-1}}{2^{d} \Gamma((d+1) / 2)},
\end{aligned}
$$

where the last inequality holds for all $L>10$.

It follows similarly to the end of the proof of Lemma 5.3 that

$$
\mathbb{P}\left(G_{u}\right)>1-\exp \left(-\lambda L \frac{\pi^{d / 2-1}}{2^{d} \Gamma((d+1) / 2)}\right) \geq 1-\frac{2^{d} \Gamma((d+1) / 2)}{\lambda L \pi^{d / 2-1}},
$$

which is larger than $3 / 4$ whenever (5.15) holds. We conclude that for such values of $\lambda$ there is a positive probability that $B^{o}(1 / 2)$ belongs to an unbounded connected component of $\mathcal{C}\left(\Pi^{\lambda}\right)$, and therefore $\lambda \geq \lambda_{c, r}$.

Acknowledgements The author would like to thank the two anonymous referees for many valuable comments and suggestions. The author would also like to thank Ronald Meester for comments on an earlier version of the paper.

Funding Open access funding provided by University of Gothenburg.

Open Access This article is licensed under a Creative Commons Attribution 4.0 International License, which permits use, sharing, adaptation, distribution and reproduction in any medium or format, as long as you give appropriate credit to the original author(s) and the source, provide a link to the Creative Commons licence, and indicate if changes were made. The images or other third party material in this article are included in the article's Creative Commons licence, unless indicated otherwise in a credit line to the material. If material is not included in the article's Creative Commons licence and your intended use is not permitted by statutory regulation or exceeds the permitted use, you will need to obtain permission directly from the copyright holder. To view a copy of this licence, visit http://creativecommons.org/licenses/by/4.0/.

\section{Appendix A}

The purpose of this appendix is to provide detailed proofs of the key lemmas used in Sects. 4 and 5, i.e. Lemmas 4.1, 5.1 and 5.2. We will start with Lemma 4.1 as this is easy. The proofs 
of the other two lemmas go through several steps, and we therefore put these into separate subsections.

\section{A.1 Measure Estimates}

Here, and in the rest of the appendix, we will let $I(\cdot)$ denote an indicator function.

Proof of Lemma 4.1 We have that

$$
\begin{aligned}
& \mu_{\lambda}\left((x, p) \in \mathbb{R}^{d} \times \mathbb{S}: \ell_{x, p, L} \cap B(o, \rho) \neq \emptyset\right) \\
& \quad=\int_{\mathbb{S}} \int_{\mathbb{R}^{d}} I\left(\ell_{x, p, L} \cap B(o, \rho)\right) \mathrm{d} x \Theta(\mathrm{d} p)=\int_{\mathbb{S}} \int_{\mathbb{R}^{d}} I\left(\ell_{o, p, L} \cap B(x, \rho)\right) \mathrm{d} x \Theta(\mathrm{d} p) \\
& =\int_{\mathbb{S}} \operatorname{Vol}\left(S_{o, p, L}(\rho)\right) \Theta(\mathrm{d} p)=\operatorname{Vol}\left(S_{o, p, L}(\rho)\right) \Theta(\mathbb{S})=\operatorname{Vol}\left(S_{o, p, L}(\rho)\right),
\end{aligned}
$$

where the second equality follows by translation invariance. By construction, the volume of $S_{o, p, L}(\rho)$ equals the volume of a $(d-1)$-dimensional ball of radius $\rho$ times $L$, plus the volume of the two "tips" which together equals that of a $d$-dimensional ball of radius $\rho$. That is,

$$
\begin{aligned}
\operatorname{Vol}\left(S_{o, p, L}(\rho)\right) & =L \operatorname{Vol}\left(B_{d-1}(o, \rho)\right)+\operatorname{Vol}\left(B_{d}(o, \rho)\right) \\
& =L \frac{\pi^{(d-1) / 2}}{\Gamma((d+1) / 2)} \rho^{d-1}+\frac{\pi^{d / 2}}{\Gamma(d / 2+1)} \rho^{d},
\end{aligned}
$$

as desired.

\section{A.2 Proof of Lemma 5.1}

In order to prove Lemma 5.1, we will go through two steps. The first of these is the following lemma which provides a lower bound on the measure of line segments that hit a given ball of radius $\rho$.

Lemma A.1 (Lower bound on line segment-ball hitting measure) Let $d \geq 2, L>0$ and $0<\rho<L / 8$. Then, for any $x \in \mathbb{R}^{d}$ with $\rho<\|x\|<L / 2-\rho$, we have that

$$
\int_{\mathbb{S}} I\left(\ell_{x, p, L} \cap B(o, \rho) \neq \emptyset\right) \mathcal{H}(\mathrm{d} p) \geq \frac{\Gamma(d / 2)}{\sqrt{\pi} \Gamma((d+1) / 2)} \frac{\rho^{d-1}}{\|x\|^{d-1}} .
$$

Remark We will only use Lemma A.1 for $0<\rho \leq 2$. However, we chose to state it more generally as it is essentially a more exact version of the lower bound of Lemma 3.1 of [11], which has been used many times (for instance in [1]).

We also note that it is possible to derive a weaker version of Lemma 4.1 from Lemma A.1 by integrating over a suitable subset of $\mathbb{R}^{d}$.

Proof By rotational invariance of the model, we may without loss of generality assume that $x=\left(x_{1}, 0, \ldots, 0\right)$ where $x_{1}>\rho$. As in Sect. 5.1 we let

$$
\ell_{x, p, \infty}=\{x+t p:-\infty<t<\infty\},
$$

and $p$ is as usual a vector such that $\|p\|=1$. Then, consider the distance between a point $\ell_{x, p, \infty}(t)$ on the line $\ell_{x, p, \infty}$ and the origin $o$

$$
\left\|\ell_{x, p, \infty}(t)-o\right\|^{2}=\|x+t p\|^{2}=\|x\|^{2}+t^{2}\|p\|^{2}+2 t\langle x, p\rangle=\|x\|^{2}+t^{2}+2 t\langle x, p\rangle
$$


which is minimized when $t=-\langle x, p\rangle$, so that

$$
\operatorname{dist}\left(\ell_{x, p, \infty}, o\right)^{2}=\|x\|^{2}+\langle x, p\rangle^{2}-2\langle x, p\rangle^{2}=\|x\|^{2}-\langle x, p\rangle^{2} .
$$

Using that $x=\left(x_{1}, 0, \ldots, 0\right)$ we then see that

$$
\begin{aligned}
& \operatorname{dist}\left(\ell_{x, p, \infty}, o\right)^{2} \leq \rho^{2} \Leftrightarrow x_{1}^{2}-x_{1}^{2} p_{1}^{2} \leq \rho^{2} \Leftrightarrow p_{1}^{2} \\
& \geq \frac{x_{1}^{2}-\rho^{2}}{x_{1}^{2}} .
\end{aligned}
$$

We note that if $x_{1} \leq \rho$, then (A.1) holds for any $p$ as indeed it should since $x$ is then inside of the ball $B(o, \rho)$. This is why we include $\|x\|>\rho$ in the assumption. Furthermore, the line segment $\ell_{x, p, L}$ has finite length $L$, which is why we need to assume that $x_{1}=\|x\|<L / 2-\rho$ in order for $\ell_{x, p, L}$ to reach the ball $B(o, \rho)$. To see that this suffices, we observe that

$$
\left\|\ell_{x, p, \infty}(-\langle x, p\rangle)-x_{1}\right\| \leq \operatorname{dist}\left(\ell_{x, p, \infty}, o\right)+x_{1}<L / 2,
$$

whenever (A.1) holds (since $-\langle x, p\rangle$ minimized the distance between $\ell_{x, p, \infty}$ and $o$ ). It follows that for every $\rho<x_{1}<L / 2-\rho$, we have that $\ell_{x, p, L} \cap B(o, \rho) \neq \emptyset$ if and only if $p_{1}^{2} \geq \frac{x_{1}^{2}-\rho^{2}}{x_{1}^{2}}$. Thus, for fixed $\rho<x_{1}<L / 2-\rho$, we get that

$$
\begin{aligned}
& \int_{\mathbb{S}} I\left(\ell_{x, p, L} \cap B(o, \rho) \neq \emptyset\right) \mathcal{H}(\mathrm{d} p) \\
& \quad=\int_{\mathbb{S}} I\left(\operatorname{dist}\left(\ell_{x, p, \infty}, o\right)^{2} \leq \rho^{2}\right) \mathcal{H}(\mathrm{d} p)=\int_{\mathbb{S}} I\left(p_{1}^{2} \geq 1-\frac{\rho^{2}}{x_{1}^{2}}\right) \mathcal{H}(\mathrm{d} p) .
\end{aligned}
$$

This is simply twice (by symmetry of $p$ and $-p$ ) the surface area (or rather the $(d-1)$ dimensional Hausdorff measure) of the spherical cap of height

$$
h=1-\sqrt{1-\frac{\rho^{2}}{x_{1}^{2}}},
$$

normalized (because of (2.6)) by the surface area of $\mathbb{S}$, which is $\frac{2 \pi^{d / 2}}{\Gamma(d / 2)}$. It is known (see [5]), that the surface area of this spherical cap equals

$$
\frac{\pi^{d / 2}}{\Gamma(d / 2)} J_{2 h-h^{2}}\left(\frac{d-1}{2}, \frac{1}{2}\right)=\frac{\pi^{d / 2}}{\Gamma(d / 2)} J_{\rho^{2} / x_{1}^{2}}\left(\frac{d-1}{2}, \frac{1}{2}\right),
$$

where $J$ is the regularized incomplete Beta function. Furthermore,

$$
\begin{aligned}
& J_{\rho^{2} / x_{1}^{2}}\left(\frac{d-1}{2}, \frac{1}{2}\right) \\
& =\frac{\int_{0}^{\rho^{2} / x_{1}^{2}} t^{(d-1) / 2-1}(1-t)^{1 / 2-1} \mathrm{~d} t}{\int_{0}^{1} t^{(d-1) / 2-1}(1-t)^{1 / 2-1} \mathrm{~d} t}=\frac{\Gamma(d / 2)}{\sqrt{\pi} \Gamma((d-1) / 2)} \int_{0}^{\rho^{2} / x_{1}^{2}} t^{(d-1) / 2-1}(1-t)^{-1 / 2} \mathrm{~d} t \\
& \geq \frac{\Gamma(d / 2)}{\sqrt{\pi} \Gamma((d-1) / 2)} \int_{0}^{\rho^{2} / x_{1}^{2}} t^{(d-1) / 2-1} \mathrm{~d} t=\frac{2 \Gamma(d / 2)}{(d-1) \sqrt{\pi} \Gamma((d-1) / 2)}\left[t^{(d-1) / 2}\right]_{0}^{\rho^{2} / x_{1}^{2}} \\
& =\frac{2 \Gamma(d / 2)}{(d-1) \sqrt{\pi} \Gamma((d-1) / 2)} \frac{\rho^{d-1}}{x_{1}^{d-1}}=\frac{\Gamma(d / 2)}{\sqrt{\pi} \Gamma((d+1) / 2)} \frac{\rho^{d-1}}{x_{1}^{d-1}},
\end{aligned}
$$


where the first equality is simply the definition of $J_{\rho^{2} / x_{1}^{2}}\left(\frac{d-1}{2}, \frac{1}{2}\right)$, where the last equality uses the identity $z \Gamma(z)=\Gamma(1+z)$ with $z=(d-1) / 2$, and where we used that

$$
\int_{0}^{1} t^{(d-1) / 2-1}(1-t)^{-1 / 2} d t=\frac{\sqrt{\pi} \Gamma((d-1) / 2)}{\Gamma(d / 2)}
$$

for $d \geq 2$. We then see that by (A.2) and (2.6),

$$
\begin{aligned}
& \int_{\mathbb{S}} I\left(\ell_{x, p, L} \cap B(o, \rho) \neq \emptyset\right) \mathcal{H}(\mathrm{d} p)=\int_{\mathbb{S}} I\left(p_{1}^{2} \geq 1-\frac{\rho^{2}}{x_{1}^{2}}\right) \mathcal{H}(\mathrm{d} p) \\
& \quad=2 \frac{\Gamma(d / 2)}{2 \pi^{d / 2}} \frac{\pi^{d / 2}}{\Gamma(d / 2)} J_{\rho^{2} / x_{1}^{2}}\left(\frac{d-1}{2}, \frac{1}{2}\right) \geq \frac{\Gamma(d / 2)}{\sqrt{\pi} \Gamma((d+1) / 2)} \frac{\rho^{d-1}}{x_{1}^{d-1}},
\end{aligned}
$$

and so for general $\rho<\|x\|<L / 2-\rho$ we get that

$$
\int_{\mathbb{S}} I\left(\ell_{x, p, L} \cap B(o, \rho) \neq \emptyset\right) \mathcal{H}(\mathrm{d} p) \geq \frac{\Gamma(d / 2)}{\sqrt{\pi} \Gamma((d+1) / 2)} \frac{\rho^{d-1}}{\|x\|^{d-1}}
$$

as required.

Our next step is to obtain a lower bound on the $\mu_{\lambda}$-measure of the set of points $(x, p) \in$ $\mathbb{R}^{d} \times \mathbb{S}$ such that their corresponding line segments $\ell_{x, p, L}$ intersect two balls $B(\gamma, 2)$ and $B(\zeta, 2)$, placed so that their centers $\gamma, \zeta$ belong to the horizontal axis. To that end, we will need the following notation. Recall $x^{r}$ defined in (2.2) and let

$$
\begin{aligned}
& \ell_{L /(32 \sqrt{d})}:=\left\{x \in \mathbb{R}^{d}:\left|x_{1}\right| \leq \frac{L}{32 \sqrt{d}} \text { and } x_{k}=0 \text { for } k=2, \ldots, d\right\}, \\
& S_{L /(32 \sqrt{d})}:=\ell_{L /(32 \sqrt{d})}^{+2} \text { and } \\
& \mathrm{Cyl}_{L /(32 \sqrt{d})}:=\left\{x \in \mathbb{R}^{d}:\left|x_{1}\right| \leq \frac{L}{32 \sqrt{d}} \text { and }\left|x^{0}\right| \leq 2 \text { for } k=2, \ldots, d\right\} .
\end{aligned}
$$

We see that $\ell_{L /(32 \sqrt{d})}$ is a horizontal line segment of length $\frac{L}{16 \sqrt{d}}$ while $S_{L /(32 \sqrt{d})}$ is the corresponding stick of radius 2 and $\mathrm{Cyl}_{L /(32 \sqrt{d})}$ is the truncation of $S_{L /(32 \sqrt{d})}$ where the tips have been removed. In order to obtain the required lower bound, we will restrict our attention to $x \in S_{L /(32 \sqrt{d})}$. We remark that $S_{L /(32 \sqrt{d})}$ will only serve as a set in which we are looking for centers $x$ of line segments $\ell_{x, p, L}$. It just happens to be a stick of radius 2 .

Recall the notation (5.5).

Lemma A.2 (Measure of line segments connecting two balls) Let $d \geq 2$, and let $\Theta(\mathrm{d} p)=$ $\phi(p) \mathcal{H}(\mathrm{d} p)$ where $\phi(p)$ satisfies (3.1) for some $\delta>0$. Furthermore, let $L>32$, and let $r_{1}, r_{2} \geq 0$ be such that $\frac{L}{8}<\min \left(r_{1}, r_{2}\right) \leq \max \left(r_{1}, r_{2}\right)<\frac{L}{\sqrt{6}}$. Then,

$$
\mu_{\lambda}\left((x, p): x \in S_{L /(32 \sqrt{d})}, B\left(-r_{1} e_{1}, 2\right) \stackrel{\ell_{x, p, L}}{\longleftrightarrow} B\left(r_{2} e_{1}, 2\right)\right) \geq \lambda \delta c_{d} L^{-d+2},
$$

where we may take

$$
c_{d}=2^{5(d-2)} \pi^{d / 2-2} \frac{1}{\sqrt{d}} \frac{\Gamma(d / 2)^{3}}{\Gamma(2 d-1)} .
$$




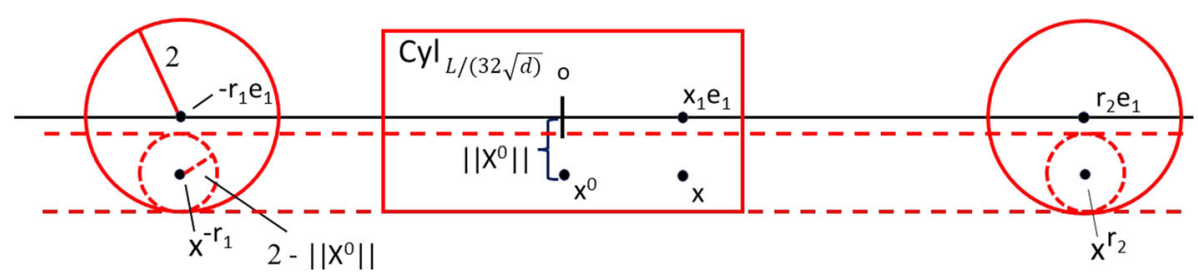

Fig. 5 An illustration of the argument leading up to (A.5). The next step is to translate the picture so that $x$ is mapped to the origin $o$

Remarks It is easy to make the statement somewhat more general. In particular, one could consider more general widths of the cylinder and ease the requirements on $r_{1}, r_{2}$. However, in contrast to Lemma A.1 we do not anticipate a wider use of this lemma, and therefore we prefer to keep it as simple as possible.

In the statement we consider $(x, p)$ such that $x \in S_{L /(32 \sqrt{d})}$, but the proof will restrict this further to $x \in \mathrm{Cyl}_{L /(32 \sqrt{d})}$. We chose to state it in the current way as this is how it will be used.

Proof We start by considering the case $x=o$. Let $r=\max \left(r_{1}, r_{2}\right)$ and observe that by symmetry,

$$
\left\{B\left(-r_{1} e_{1}, 2\right) \stackrel{\ell_{o, p, L}}{\longleftrightarrow} B\left(r_{2} e_{1}, 2\right)\right\}=\left\{B\left(-r e_{1}, 2\right) \stackrel{\ell_{o, p, L}}{\longleftrightarrow} B\left(r e_{1}, 2\right)\right\},
$$

since here we used $x=o$. Furthermore, we clearly have that

$$
\left\{B\left(-r e_{1}, 2\right) \stackrel{\ell_{o, p, L}}{\longleftrightarrow} B\left(r e_{1}, 2\right)\right\}=\left\{\ell_{o, p, L} \cap B\left(r e_{1}, 2\right) \neq \emptyset\right\},
$$

since any $\ell_{o, p, L}$ touching $B\left(r e_{1}, 2\right)$ must also touch $B\left(-r e_{1}, 2\right)$ (again by symmetry). We therefore conclude that

$$
\begin{aligned}
& \int_{\mathbb{S}} I\left(B\left(-r_{1} e_{1}, 2\right) \stackrel{\ell_{o, p, L}}{\longleftrightarrow} B\left(r_{2} e_{1}, 2\right)\right) \mathcal{H}(\mathrm{d} p) \\
& \quad=\int_{\mathbb{S}} I\left(\ell_{o, p, L} \cap B\left(r e_{1}, 2\right) \neq \emptyset\right) \mathcal{H}(\mathrm{d} p)=\int_{\mathbb{S}} I\left(\ell_{r e_{1}, p, L} \cap B(o, 2) \neq \emptyset\right) \mathcal{H}(\mathrm{d} p) \\
& \quad \geq \frac{\Gamma(d / 2)}{\sqrt{\pi} \Gamma((d+1) / 2)} \frac{2^{d-1}}{r^{d-1}}=\frac{\Gamma(d / 2)}{\sqrt{\pi} \Gamma((d+1) / 2)}\left(\frac{2}{\max \left(r_{1}, r_{2}\right)}\right)^{d-1}
\end{aligned}
$$

by translation invariance, and by using Lemma A.1 with $\rho=2$, which we can since $L>$ $16=8 \rho$ and

$$
2<\frac{L}{8}<\left\|r e_{1}\right\|<\frac{L}{\sqrt{6}} \leq \frac{L}{2}-2,
$$

by the assumption that $L>32$.

Next, we consider general $x \in \mathrm{Cyl}_{L /(32 \sqrt{d})}$, and the idea is to reduce this general case to the simpler first case where $x=o$ (see also Fig. 5).

As we will see, this can be done by translation and by replacing the balls $B\left(-r_{1} e_{1}, 2\right)$ with smaller ones (i.e. $\left.B\left(x^{-r_{1}}, 2-\left\|x^{0}\right\|\right)\right)$ whose centers are more conveniently placed. To this end, observe that

$$
B\left(x^{-r_{1}}, 2-\left\|x^{0}\right\|\right) \subset B\left(-r_{1} e_{1}, 2\right) \text { and } B\left(x^{r_{2}}, 2-\left\|x^{0}\right\|\right) \subset B\left(r_{2} e_{1}, 2\right) .
$$


Indeed, let $y \in B\left(x^{-r_{1}}, 2-\left\|x^{0}\right\|\right)$ and note that

$$
\begin{aligned}
\left\|-r_{1} e_{1}-y\right\| & \leq\left\|-r_{1} e_{1}-x^{-r_{1}}\right\|+\left\|x^{-r_{1}}-y\right\|=\left\|x^{0}\right\|+\left\|x^{-r_{1}}-y\right\| \\
& \leq\left\|x^{0}\right\|+2-\left\|x^{0}\right\|=2 .
\end{aligned}
$$

Therefore,

$$
\left\{B\left(x^{-r_{1}}, 2-\left\|x^{0}\right\|\right) \stackrel{\ell_{x, p, L}}{\longleftrightarrow} B\left(x^{r_{2}}, 2-\left\|x^{0}\right\|\right)\right\} \subset\left\{B\left(-r_{1} e_{1}, 2\right) \stackrel{\ell_{x, p, L}}{\longleftrightarrow} B\left(r_{2} e_{1}, 2\right)\right\},
$$

and so

$$
\begin{aligned}
& \int_{\mathbb{S}} I\left(B\left(-r_{1} e_{1}, 2\right) \stackrel{\ell_{x, p, L}}{\longleftrightarrow} B\left(r_{2} e_{1}, 2\right)\right) \mathcal{H}(\mathrm{d} p) \\
& \geq \int_{\mathbb{S}} I\left(B\left(x^{-r_{1}}, 2-\left\|x^{0}\right\|\right) \stackrel{\ell_{x, p, L}}{\longleftrightarrow} B\left(x^{r_{2}}, 2-\left\|x^{0}\right\|\right)\right) \mathcal{H}(\mathrm{d} p) \\
& =\int_{\mathbb{S}} I\left(B\left(x^{-r_{1}}-x, 2-\left\|x^{0}\right\|\right) \stackrel{\ell_{o, p, L}}{\longleftrightarrow} B\left(x^{r_{2}}-x, 2-\left\|x^{0}\right\|\right)\right) \mathcal{H}(\mathrm{d} p) \\
& =\int_{\mathbb{S}} I\left(B\left(-\left(r_{1}+x_{1}\right) e_{1}, 2-\left\|x^{0}\right\|\right) \stackrel{\ell_{o, p, L}}{\longleftrightarrow} B\left(\left(r_{2}-x_{1}\right) e_{1}, 2-\left\|x^{0}\right\|\right)\right) \mathcal{H}(\mathrm{d} p) \\
& \geq \frac{\Gamma(d / 2)}{\sqrt{\pi} \Gamma((d+1) / 2)}\left(\frac{2-\left\|x^{0}\right\|}{\max \left(r_{1}+x_{1}, r_{2}-x_{1}\right)}\right)^{d-1}
\end{aligned}
$$

where the first equality follows by translation invariance (translating $x$ to $o$ ), and the second inequality follows by using Lemma A.1 with $\rho=2-\left\|x^{0}\right\|$. As before, we may use this lemma since by assumption

$$
\max \left(r_{1}+x_{1}, r_{2}-x_{1}\right) \geq \min \left(r_{1}, r_{2}\right)-\left|x_{1}\right|>\frac{L}{8}-\frac{L}{32 \sqrt{d}}>2 \geq 2-\left\|x^{0}\right\|=\rho,
$$

and

$$
\max \left(r_{1}+x_{1}, r_{2}-x_{1}\right) \leq \max \left(r_{1}, r_{2}\right)+\frac{L}{32 \sqrt{d}}<\frac{L}{\sqrt{6}}+\frac{L}{32 \sqrt{d}}<\frac{L}{2}-2,
$$

since $L>32$. Clearly we also have that $L>8\left(2-\left\|x^{0}\right\|\right)=8 \rho$. Furthermore, since $\max \left(r_{1}+x_{1}, r_{2}-x_{1}\right)<L / 2$, it follows from (A.6) that

$$
\int_{\mathbb{S}} I\left(B\left(-r_{1} e_{1}, 2\right) \stackrel{\ell_{x, p, L}}{\longleftrightarrow} B\left(r_{2} e_{1}, 2\right)\right) \mathcal{H}(\mathrm{d} p) \geq \frac{\Gamma(d / 2)}{\sqrt{\pi} \Gamma((d+1) / 2)} 2^{d-1}\left(\frac{2-\left\|x^{0}\right\|}{L}\right)^{d-1} .
$$


We can now integrate over $x \in \mathrm{Cyl}_{L /(32 \sqrt{d})}$ to obtain

$$
\begin{aligned}
& \mu_{\lambda}\left((x, p): x \in \mathrm{Cyl}_{L /(32 \sqrt{d})}, B\left(-r_{1} e_{1}, 2\right) \stackrel{\ell_{x, p, L}}{\longleftrightarrow} B\left(r_{2} e_{1}, 2\right)\right) \\
& =\lambda \int_{\mathrm{Cyl}_{L /(32 \sqrt{d})}} \int_{\mathbb{S}} I\left(B\left(-r_{1} e_{1}, 2\right) \stackrel{\ell_{x, p, L}}{\longleftrightarrow} B\left(r_{2} e_{1}, 2\right)\right) \Theta(\mathrm{d} p) \mathrm{d} x \\
& \geq \lambda \int_{\mathrm{Cyl}_{L /(32 \sqrt{d})}} \int_{\mathbb{S}} I\left(B\left(-r_{1} e_{1}, 2\right) \stackrel{\ell_{x, p, L}}{\longleftrightarrow} B\left(r_{2} e_{1}, 2\right)\right) \delta \mathcal{H}(\mathrm{d} p) \mathrm{d} x \\
& \geq \lambda \delta \frac{2^{d-1} \Gamma(d / 2)}{\sqrt{\pi} \Gamma((d+1) / 2)} \int_{\mathrm{Cyl}_{L /(32 \sqrt{d})}}\left(\frac{2-\left\|x^{0}\right\|}{L}\right)^{d-1} \mathrm{~d} x \\
& =\lambda \delta \frac{2^{d-1} \Gamma(d / 2)}{\sqrt{\pi} \Gamma((d+1) / 2)} \frac{L}{32 \sqrt{d}} L^{-d+1} \int_{\|y\| \leq 2}(2-\|y\|)^{d-1} \mathrm{~d} y
\end{aligned}
$$

where the last integral is for $y \in \mathbb{R}^{d-1}$. We therefore have that

$$
\begin{aligned}
& \int_{\|y\| \leq 2}(2-\|y\|)^{d-1} \mathrm{~d} y \\
& =\frac{2 \pi^{(d-1) / 2}}{\Gamma((d-1) / 2)} \int_{0}^{2}(2-r)^{d-1} r^{d-2} \mathrm{~d} r=\frac{2 \pi^{(d-1) / 2}}{\Gamma((d-1) / 2)} \frac{2^{2(d-1)} \Gamma(d-1) \Gamma(d)}{\Gamma(2 d-1)}
\end{aligned}
$$

so that finally

$$
\begin{aligned}
& \mu_{\lambda}\left((x, p): x \in \mathrm{Cyl}_{L /(32 \sqrt{d})}, B\left(-r_{1} e_{1}, 2\right) \stackrel{\ell_{x, p, L}}{\longleftrightarrow} B\left(r_{2} e_{1}, 2\right)\right) \\
& \geq \lambda \delta \frac{L}{32 \sqrt{d}} L^{-d+1} \frac{2^{d-1} \Gamma(d / 2)}{\sqrt{\pi} \Gamma((d+1) / 2)} \frac{2 \pi^{(d-1) / 2}}{\Gamma((d-1) / 2)} \frac{2^{2(d-1)} \Gamma(d-1) \Gamma(d)}{\Gamma(2 d-1)} \\
& =\lambda \delta L^{-d+2} \frac{\Gamma(d) 2^{1-d} \sqrt{\pi}}{\Gamma((d+1) / 2)} \frac{\Gamma(d-1) 2^{1-(d-1)} \sqrt{\pi}}{\Gamma((d-1) / 2)} \frac{2^{5(d-1)}}{32} \frac{1}{\sqrt{d}} \pi^{d / 2-2} \frac{\Gamma(d / 2)}{\Gamma(2 d-1)} \\
& =\lambda \delta L^{-d+2} \Gamma(d / 2) \Gamma(d / 2) 2^{5(d-2)} \frac{1}{\sqrt{d}} \pi^{d / 2-2} \frac{\Gamma(d / 2)}{\Gamma(2 d-1)} \\
& =\lambda \delta 2^{5(d-2)} \frac{1}{\sqrt{d}} \pi^{d / 2-2} \frac{\Gamma(d / 2)^{3}}{\Gamma(2 d-1)} L^{-d+2},
\end{aligned}
$$

by using the identity $\Gamma(z) \Gamma(z+1 / 2)=2^{1-2 z} \sqrt{\pi} \Gamma(2 z)$ for $z=d / 2$ and $z=(d-1) / 2$ in the second to last equality.

Recall the notation $R^{-16}\left(D^{u}\right)$ from Sect. 5. We are now ready to prove Lemma 5.1.

Proof of Lemma 5.1 We will use Lemma A.2 and need to make preparations for this. To that end, consider

$$
\ell_{\gamma, \zeta}:=\{\gamma+t(\zeta-\gamma): 0 \leq t \leq 1\}
$$

i.e. the line segment between $\gamma$ and $\zeta$. Then, let $z^{*} \in \ell_{\gamma, \zeta}$ be such that $z_{1}^{*}=-L / 4$ so that $z^{*}$ is the point on the line $\ell_{\gamma, \zeta}$ whose first coordinate is in the middle of the right-hand side and the left-hand side of $D^{(-1,0)}$. Next, we let

$$
\ell^{*}:=\left\{z \in \ell_{\gamma, \zeta}:\left\|z-z^{*}\right\| \leq \frac{L}{32 \sqrt{d}}\right\},
$$




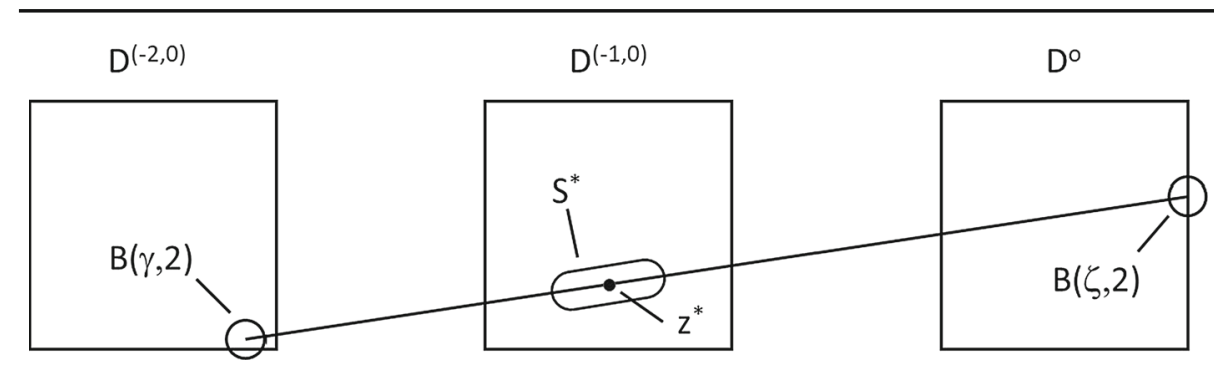

Fig. 6 The figure depicts the balls $B(\gamma, 2)$ where $\gamma \in D^{(-2,0)}$ and $B(\zeta, 2)$ where $\zeta \in R^{-16}\left(D^{o}\right)$. We can also see $z^{*} \in \ell_{\gamma, \zeta}$ and $S^{*}$. Note that the region $S^{*}$ will typically not be centered in the middle between $\gamma, \zeta$. The figure is not drawn to scale

and $S^{*}:=\left(\ell^{*}\right)^{+2}$ (see Fig. 6 for a depiction).

Clearly, the triple $S^{*}, B(\gamma, 2)$ and $B(\zeta, 2)$ is just a rotated and translated version of the triple $S_{L /(32 \sqrt{d})}, B\left(-r_{1} e_{1}, 2\right)$ and $B\left(r_{2} e_{1}, 2\right)$ of the statement of Lemma A.2. In order to use that lemma, we need that $L>32$ which holds by assumption, and in addition we need to verify that

$$
\frac{L}{8}<\min \left(\left\|z^{*}-\gamma\right\|,\left\|z^{*}-\zeta\right\|\right) \leq \max \left(\left\|z^{*}-\gamma\right\|,\left\|z^{*}-\zeta\right\|\right)<\frac{L}{\sqrt{6}} .
$$

We observe that it follows from the definition of $D^{u}$, i.e. (5.4), that for any $z \in D^{(-1,0)}$,

$$
\|z-\gamma\|^{2} \leq\left(\frac{L}{4}+\frac{L}{8 \sqrt{d}}\right)^{2}+(d-1) \frac{L^{2}}{64 d}=\frac{L^{2}}{16}+\frac{L^{2}}{16 \sqrt{d}}+\frac{L^{2}}{64}<\frac{L^{2}}{6},
$$

for every $d \geq 2$. Here, we used that $D^{u}$ has side length $\frac{L}{8 \sqrt{d}}$, and that the distance between the centers of two neighboring boxes is $L / 4$. Furthermore, it is easy to see that

$$
\|z-\gamma\| \geq \frac{L}{4}-\frac{L}{8 \sqrt{d}}>\frac{L}{8}
$$

for $d \geq 2$. Clearly (A.8) and (A.9) must also hold for $\zeta$ in place of $\gamma$ and so (A.7) follows since $z^{*} \in D^{(-1,0)}$.

Next, we need to show that

$$
S^{*} \subset D^{(-1,0)}
$$

since then we can conclude that

$$
\begin{aligned}
& \mu_{\lambda}\left((x, p): x \in D^{(-1,0)}, B(\gamma, 2) \stackrel{\ell_{x, p, L}}{\longleftrightarrow} B(\zeta, 2)\right) \\
& \geq \mu_{\lambda}\left((x, p): x \in S^{*}, B(\gamma, 2) \stackrel{\ell_{x, p, L}}{\longleftrightarrow} B(\zeta, 2)\right) \\
& =\lambda \int_{S^{*}} \int_{\mathbb{S}} I\left(B(\gamma, 2) \stackrel{\ell_{x, p, L}}{\longleftrightarrow} B(\zeta, 2)\right) \Theta(\mathrm{d} p) \mathrm{d} x \geq \lambda \delta c_{d} L^{-d+2},
\end{aligned}
$$

where we used Lemma A.2 with $c_{d}$ as in that lemma in the last inequality.

In order to verify (A.10), observe first that for any $z=\left(z_{1}, \ldots, z_{d}\right) \in \ell^{*}$ we have that $\left|z_{1}-z_{1}^{*}\right| \leq \frac{L}{32 \sqrt{d}}$. Furthermore, by using that $\gamma \in D^{(-2,0)}$ and that $\zeta \in R^{-16}\left(D^{o}\right)$, it 


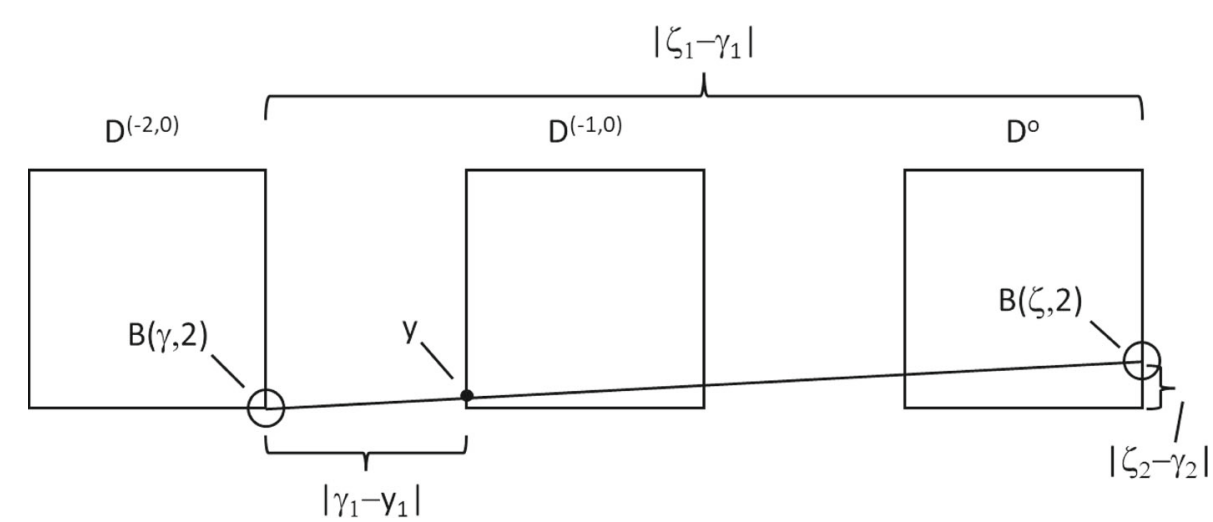

Fig. 7 The worst case is depicted, i.e. where $\gamma$ is the lower right corner of $D^{(-2,0)}$ and $\zeta \in R\left(D^{o}\right)$ is as close to the bottom of $D^{o}$ as it is allowed. The figure is not drawn to scale

is a straightforward, although tedious, trigonometric exercise (which is outlined below) to conclude that

$$
\left|z_{k}\right| \leq \frac{L}{16 \sqrt{d}}-4
$$

for $k=2, \ldots, d$, and it follows that (A.10) holds.

In order to understand why (A.12) is correct, consider the second coordinate $z_{2}$. All other coordinates except the first will play no role in this explanation and we therefore ignore these. Note that the extreme case in the sense of $z_{2}$ being as "low" as possible would be when $\gamma=$ $\left(\gamma_{1}, \ldots, \gamma_{d}\right)$ and $\zeta=\left(\zeta_{1}, \ldots, \zeta_{d}\right)$ are such that $\gamma_{1}=-\frac{L}{2}+\frac{L}{16 \sqrt{d}}, \gamma_{2}=-\frac{L}{16 \sqrt{d}}, \zeta_{1}=\frac{L}{16 \sqrt{d}}$ and $\zeta_{2}=-\frac{L}{16 \sqrt{d}}+16$ (see Fig. 7).

Then, let $y \in \ell_{\gamma, \zeta}$ be such that $y_{1}=-\frac{L}{4}-\frac{L}{16 \sqrt{d}}$. We see that $y$ is where $\ell_{\gamma, \zeta}$ intersects the left side of $D^{(-1,0)}$ and that we must have that

$$
\frac{\left|y_{2}-\gamma_{2}\right|}{\left|y_{1}-\gamma_{1}\right|}=\frac{\left|\zeta_{2}-\gamma_{2}\right|}{\left|\zeta_{1}-\gamma_{1}\right|} \text {. }
$$

We conclude that

$$
\begin{aligned}
& \left|y_{2}-\gamma_{2}\right|=\left|y_{2}-\left(-\frac{L}{16 \sqrt{d}}\right)\right| \\
& =\left|y_{1}-\gamma_{1}\right| \frac{\left|\zeta_{2}-\gamma_{2}\right|}{\left|\zeta_{1}-\gamma_{1}\right|}=\left(\frac{L}{4}-\frac{L}{8 \sqrt{d}}\right) \frac{16}{L / 2} \geq 4 .
\end{aligned}
$$

It follows that for any $z \in \ell^{*}$ we must have that

$$
z_{2} \geq-\frac{L}{16 \sqrt{d}}+4
$$

and by expanding this argument we conclude that (A.12) must hold for $k=2, \ldots, d$. It is worth noting that it is important for this argument that $\zeta \in R^{-16}\left(D^{o}\right)$ rather than just being an arbitrary point on the right-hand side of $D^{o}$. To see this, consider Fig. 7 and note that if $\zeta$ would belong to the bottom right corner of $D^{o}$, then the intersection of $\ell_{\gamma, \zeta}$ with $D^{(-1,0)}$ could be along the bottom part of $D^{(-1,0)}$. Then, $S^{*}$ would spill over the boundary of $D^{(-1,0)}$ and so (A.10) would no longer hold. 


\section{A.3 Conditions for Disjointness}

Our first lemma of this subsection is an elementary exercise, and in it we obtain the smallest distance between a parametrized infinite line and a point on another line. For this lemma and the next, let

$$
f(t, \tau)=\left\|\ell_{x, p, \infty}(t)-\ell_{y, q, \infty}(\tau)\right\|^{2}=\|x+t p-\tau q\|^{2} .
$$

Lemma A.3 (Distance from a line to a point on another line) We have that

$$
\begin{aligned}
& \operatorname{dist}\left(\ell_{x, p, \infty}(t), \ell_{y, q, \infty}\right)^{2} \\
& \quad=\|x-y\|^{2}+t^{2}\left(1-\langle p, q\rangle^{2}\right)-\langle x-y, q\rangle^{2}+2 t(\langle x-y, p\rangle-\langle p, q\rangle\langle x-y, q\rangle) .
\end{aligned}
$$

Proof We start by noting that without loss of generality, we can take $y=o$. Consider $f(t, \tau)$ from (A.13), then fix $t$ and observe that

$$
\begin{aligned}
& g(\tau)=f(t, \tau)=\|x\|^{2}+\|t p-\tau q\|^{2}+2\langle x, t p-\tau q\rangle \\
& \quad=\|x\|^{2}+t^{2}+\tau^{2}-2 t \tau\langle p, q\rangle+2 t\langle x, p\rangle-2 \tau\langle x, q\rangle,
\end{aligned}
$$

where we used that $\|p\|=\|q\|=1$. We see that $g^{\prime}(\tau)=2 \tau-2 t\langle p, q\rangle-2\langle x, q\rangle$, and setting $g^{\prime}(\tau)$ to zero we obtain $\tau=t\langle p, q\rangle+\langle x, q\rangle$. This clearly corresponds to a minimum, and inserting this into the expression for $g(\tau)$ we then obtain

$$
\begin{aligned}
& g(t\langle p, q\rangle+\langle x, q\rangle)-\|x\|^{2} \\
& =t^{2}+(t\langle p, q\rangle+\langle x, q\rangle)^{2}-2 t(t\langle p, q\rangle+\langle x, q\rangle)\langle p, q\rangle \\
& \quad+2 t\langle x, p\rangle-2(t\langle p, q\rangle+\langle x, q\rangle)\langle x, q\rangle \\
& =t^{2}\left(1-\langle p, q\rangle^{2}\right)-\langle x, q\rangle^{2}+2 t(\langle x, p\rangle-\langle p, q\rangle\langle x, q\rangle) .
\end{aligned}
$$

The result for general $y$ follows by translation.

Next, let

$$
h(t)=\operatorname{dist}\left(\ell_{x, p, \infty}(t), \ell_{y, q, \infty}\right)^{2}=\min _{\tau} f(t, \tau) .
$$

Lemma A.4 (Minimizer of distance between two lines) If $\langle p, q\rangle \neq 0$, then $h(t)$ is minimized by

$$
t_{\min }=-\frac{\langle x-y, p\rangle-\langle p, q\rangle\langle x-y, q\rangle}{1-\langle p, q\rangle^{2}}
$$

and furthermore, for any $a \in \mathbb{R}$,

$$
h\left(t_{\min }+a\right)=h\left(t_{\min }\right)+a^{2}\left(1-\langle p, q\rangle^{2}\right) .
$$

Proof By Lemma A.3 we have that

$$
h^{\prime}(t)=2 t\left(1-\langle p, q\rangle^{2}\right)+2(\langle x-y, p\rangle-\langle p, q\rangle\langle x-y, q\rangle),
$$

and since $h^{\prime \prime}(t)>0$ if $\langle p, q\rangle \neq 0$, the function $h(t)$ is minimized when $h^{\prime}(t)=0$, from which the first statement follows. We then see that

$$
\begin{aligned}
& h\left(t_{\min }+a\right)=\|x-y\|^{2} \\
& \quad+\left(t_{\min }+a\right)^{2}\left(1-\langle p, q\rangle^{2}\right)-\langle x-y, q\rangle^{2}+2\left(t_{\min }+a\right)(\langle x-y, p\rangle-\langle p, q\rangle\langle x-y, q\rangle) \\
& \quad=h\left(t_{\min }\right)+a^{2}\left(1-\langle p, q\rangle^{2}\right)+2 a t_{\min }\left(1-\langle p, q\rangle^{2}\right)+2 a(\langle x-y, p\rangle-\langle p, q\rangle\langle x-y, q\rangle) \\
& \quad=h\left(t_{\min }\right)+a^{2}\left(1-\langle p, q\rangle^{2}\right),
\end{aligned}
$$


by using the expression for $t_{\min }$.

Proof of Lemma 5.2 Let $t_{\min }$ be as in in the statement of Lemma A.4 and observe that by that same lemma,

$$
h\left(t_{\min }+a\right)=h\left(t_{\min }\right)+a^{2}\left(1-\langle p, q\rangle^{2}\right) \geq a^{2} \frac{1}{2},
$$

by our assumption on $|\langle p, q\rangle|$. Let $t_{1}, \tau_{1}$ be such that (5.7) holds (i.e. $\| \ell_{x, p, \infty}\left(t_{1}\right)-$ $\left.\ell_{y, q, \infty}\left(\tau_{1}\right) \| \leq 2\right)$. We can then conclude that $t_{1} \in\left(t_{\min }-2 \sqrt{2}, t_{\min }+2 \sqrt{2}\right)$, since for any $t \notin\left(t_{\min }-2 \sqrt{2}, t_{\min }+2 \sqrt{2}\right)$, we have that $h(t) \geq \frac{(2 \sqrt{2})^{2}}{2}=4$ by the observation above. Furthermore we see that for any $t \notin\left(t_{\min }-6 \sqrt{2}, t_{\min }+6 \sqrt{2}\right)$

$$
h(t) \geq(6 \sqrt{2})^{2} \frac{1}{2}=36 .
$$

It follows that if $\left|t-t_{1}\right| \geq 6 \sqrt{2}+2 \sqrt{2}=8 \sqrt{2}$, then $h(t) \geq 36$ so that for any such $t$,

$$
\left\|\ell_{x, p, \infty}(t)-\ell_{y, q, \infty}(\tau)\right\| \geq 6
$$

for every $\tau$. Finally, we simply observe that $8 \sqrt{2} \leq 12$.

\section{References}

1. Broman, E.I., Tykesson, J.: Connectedness of Poisson cylinders in Euclidean space. Ann. Inst. Henri Poincaré Probab. Stat. 52(1), 102-126 (2016)

2. Domany, E., Kinzel, W.: Equivalence of cellular automata to Ising models and directed percolation. Phys. Rev. Lett. 53(4), 311-314 (1984)

3. Durrett, R.: Oriented Percolation in two dimensions. Ann. Probab. 12(4), 999-1040 (1984)

4. Hall, P.: On continuum percolation. Ann. Probab. 13(4), 1250-1266 (1985)

5. Li, S.: Concise formulas for the area and volume of a hyperspherical cap. Asian J. Math. Stat. 4(1), 66-70 (2011)

6. Liggett, T.M.: Survival of discrete time growth models, with applications to oriented Percolation. Ann. Appl. Probab. 5(3), 613-636 (1995)

7. Meester, R., Roy, R.: Continuum Percolation. Cambridge University Press, Cambridge (1996)

8. Mietta, J.L., Negri, R.M., Tamborenea, P.I.: Numerical simulations of stick percolation: application to the study of structured magnetorheological elastomers. J. Phys. Chem. C 118(35), 20594-20604 (2014)

9. Roy, R.: Percolation of poisson sticks on the plane. Probab. Theory Related Fields 89(4), 503-517 (1991)

10. Tarasevich, Y.Y., Eserkepov, A.V.: Percolation thresholds for discorectangles: numerical estimation for a range of aspect ratios. Phys. Rev. E 101(2), 022108 (2020)

11. Tykesson, J., Windisch, D.: Percolation in the vacant set of Poisson cylinders. Probab. Theory Related Fields 154(1-2), 165-191 (2012)

Publisher's Note Springer Nature remains neutral with regard to jurisdictional claims in published maps and institutional affiliations. 